\title{
Laser Control of Singlet-Pairing Process in an Ultracold Spinor Mixture
}

\author{
Jianwen Jie, ${ }^{1,2}$ Yonghong Yu, ${ }^{2}$ Dajun Wang, ${ }^{3}$ and Peng Zhang ${ }^{2,4,5, *}$ \\ ${ }^{1}$ Shenzhen Institute for Quantum Science and Engineering, \\ Southern University of Science and Technology, Shenzhen, 518055, China \\ ${ }^{2}$ Department of Physics, Renmin University of China, Beijing, 100872, China \\ ${ }^{3}$ Department of Physics, The Chinese University of Hong Kong, Hong Kong SAR, China \\ ${ }^{4}$ Beijing Computational Science Research Center, Beijing, 100084, China \\ ${ }^{5}$ Beijing Key Laboratory of Opto-electronic Functional Materials \& Micro-nano Devices (Renmin University of China)
}

(Dated: today)

\begin{abstract}
In the mixture of ultracold spin-1 atoms of two different species A and B (e.g., ${ }^{23} \mathrm{Na}(\mathrm{A})$ and $\left.{ }^{87} \mathrm{Rb}(\mathrm{B})\right)$, inter-species singlet-pairing process $\mathrm{A}_{+1}+\mathrm{B}_{-1} \rightleftharpoons \mathrm{A}_{-1}+\mathrm{B}_{+1}$ can be induced by the spin-dependent inter-atomic interaction, where subscript \pm 1 denotes the magnetic quantum number. Nevertheless, one cannot isolate this process from other spin-changing processes, which are usually much stronger, by tuning the bias real magnetic field. As a result, it is difficult to clearly observe singlet-pairing process and precisely measure the corresponding interaction strength. In this work we propose to control the singlet-pairing process via combining the real magnetic field and a laser-induced species-dependent synthetic magnetic field. With our approach one can significantly enhance this process and simultaneously suppress all other spin-changing processes. We illustrate our approach for both a confined two-atom system and a binary mixture of spinor Bose-Einstein condensates. Our control scheme is helpful for the precise measurement of the weakly singlet-pairing interaction strength and the entanglement generation of two different atoms.
\end{abstract}

\section{INTRODUCTION}

In last few decades, spinor Bose-Einstein condensates (BECs) was one of the most inspiring workhorses for studying diverse physics including spin textures, topological excitation and non-equilibrium quantum dynamics $[1,2]$. In recent years, the vitality expansion of spinor BECs is well done by experimentally realizing of plentiful dramatical physical scenes, including $\mathrm{SU}(1,1)$ interferometer [3-7], quantum synchronization [8, 9], gauge invariance [10], entanglement generation [11, 12] and dynamical quantum phase transitions [13].

In a single-species spinor BEC, e.g., the BEC of spin-1 ${ }^{87} \mathrm{Rb}[14]$ or ${ }^{23} \mathrm{Na}$ [15] atoms, the spin-dependent interatomic interaction can induce a two-body spin-mixing process $\mathrm{A}_{0}+\mathrm{A}_{0} \rightleftharpoons \mathrm{A}_{+1}+\mathrm{A}_{-1}$, where $\mathrm{A}$ denotes the atomic species (e.g., ${ }^{87} \mathrm{Rb}$ or ${ }^{23} \mathrm{Na}$ ) and the subscript $0, \pm 1$ denotes the magnetic quantum number of atomic spin. This process can induce fruitful spin dynamics which were successfully observed [16-18] and can be used for the generation of spin squeezing, entanglement state as well as quantum metrology gain [3-5, 7, 19-23].

Furthermore, the binary mixture of spin-1 bosonic atoms has also been experimentally realized with several atomic combinations [10, 24-26]. In such twospecies system the spin-dependent inter-species interaction can induce various types of spin-changing processes for two different atoms, i.e., the spin-mixing processes $\mathrm{A}_{0}+\mathrm{B}_{0} \rightleftharpoons \mathrm{A}_{ \pm 1}+\mathrm{B}_{\mp 1}$ as well as the spin-exchanging processes $\mathrm{A}_{0}+\mathrm{B}_{ \pm 1} \rightleftharpoons \mathrm{A}_{ \pm 1}+\mathrm{B}_{0}$ and $\mathrm{A}_{+1}+\mathrm{B}_{-1} \rightleftharpoons \mathrm{A}_{-1}+\mathrm{B}_{+1}$, where $\mathrm{A}$ and $\mathrm{B}$ denote the atomic species and the sub-

* pengzhang@ruc.edu.cn script $0, \pm 1$ denotes the atomic magnetic quantum number, as above. These processes can induce coherent heteronuclear spin dynamics [27, 28] and significantly influence the quantum phases of the two-species spin-1 BEC [29-34]. In the experiments one can control most of the above spin-changing processes via the bias magnetic field. Explicitly, to enhance one specific process, one can just tune the magnetic field to a particular value so that the total Zeeman energy of the two atoms before this process is close to the one after this process, i.e., the initial and finial two-atom spin state of this process is near "resonant" with each other. Using this technique Li et al. successfully observed the spin-exchanging processes $\mathrm{A}_{0}+\mathrm{B}_{ \pm 1} \rightleftharpoons \mathrm{A}_{ \pm 1}+\mathrm{B}_{0}$ in the mixture of ultracold ${ }^{87} \mathrm{Rb}$ and ${ }^{23} \mathrm{Na}$ atoms [24].

The process $\mathrm{A}_{+1}+\mathrm{B}_{-1} \rightleftharpoons \mathrm{A}_{-1}+\mathrm{B}_{+1}$ is also called as the "singlet-paring process" (or "quadrupole exchange process" [26]). In this process magnetic quantum number of each atom can be changed by \pm 2 , while in all other processes $\langle 1\rangle-\langle 6\rangle$ the single-atom magnetic quantum number can only be changed by \pm 1 . However, this interesting process cannot be controlled via the the above approach. This can be explained as following. As shown below (Sec. III. A), for the singlet-paring process the above Zeemanenergy "resonant" condition can be satisfied only when the bias magnetic field is zero. Nevertheless, in this case such "resonant" condition is also satisfied for all other spin-changing processes. As a result, the singlet-paring process would be mixed with other processes and thus cannot be clearly detected. Moreover, the singlet-paring process is very weak. For instance, its strength is only $0.8 \%$ compare to the strength of other spin-exchange processes for the ${ }^{87} \mathrm{Rb}^{23} \mathrm{Na}$ mixture. Due to these facts, it is difficult to clearly observe spinglet-pairing process and precisely measure the corresponding interaction strength 
$[26]$.

In this work we propose an approach to effectively controlling the singlet-pairing process. Our basic idea is to apply both the real magnetic field and the synthetic magnetic field (SMF) induced by the vector light shift of a circular-polarized laser beam, which was found in atom physics in 1970s [35] and has been used experimentally in the field of ultracold spinor gases $[24,36]$. In this case the total "effective magnetic field" experienced by each atom would be the summation of the real magnetic field and the SMF. An important property of the SMF is that it is species-dependent. As a result, in the presence of the SMF the total "effective magnetic field" experienced by atoms of different species would be different, and can be independently controlled, as illustrated in the experiment of Ref. [24]. Therefore, one can tune the system to some points where the singlet-pairing process is energetically resonant, while the other spin-changing processes are far-off resonant. Around this point the singlet-pairing process can be significantly enhanced and isolated from other processes.

In previous experiments of ultracold ${ }^{87} \mathrm{Rb}$ and ${ }^{23} \mathrm{Na}$ atoms $[24,25]$ the SMF has been illustrated for the manipulation of the spin-exchange process $\mathrm{A}_{0}+\mathrm{B}_{-1} \rightleftharpoons$ $\mathrm{A}_{-1}+\mathrm{B}_{0}$ and the spin-mixing process $\mathrm{A}_{0}+\mathrm{B}_{0} \rightleftharpoons \mathrm{A}_{+1}+$ $\mathrm{B}_{-1}$. Nevertheless, in the absence of the SMF, these two processes can still be enhanced via real magnetic fields, with the "resonant" method mentioned above. The experiments in Refs. [24, 25] show that in the presence of the SMF the values of the real magnetic field required to enhance these two processes are shifted. For our case, as shown above, one cannot enhance the singlet-pairing process and simultaneously isolate it from other processes only with the real magnetic field. Thus the application of the SMF is necessary.

In the following sections we take the mixture of ultracold ${ }^{87} \mathrm{Rb}$ and ${ }^{23} \mathrm{Na}$ atoms as an example, and illustrate our approach for both a confined two-atom system and the binary mixture of BECs of ${ }^{87} \mathrm{Rb}$ and ${ }^{23} \mathrm{Na}$ atoms. Our approach can be used for the observation and manipulation of the singlet-paring process, the precise measurement of the corresponding interaction intensity, as well as the entanglement generation of two different spin1 ultracold atoms.

The remainder of this article is organized as follows. In Sec. II we introduce the inter-atomic interactions and related spin-changing processes of the mixture of ultracold ${ }^{87} \mathrm{Rb}$ and ${ }^{23} \mathrm{Na}$ atoms. In Sec. III our proposal for the manipulation of singlet-pairing process is introduced. In Secs. IV and V we further illustrate our proposal for a confined two-atom system and a binary mixture of BECs, respectively. A summary for our results and some discussions are given in Sec. VI. In the appendix we present some details of our calculation.

\section{SPIN-CHANGING SCATTERING PROCESS BETWEEN ULTRACOLD SPIN-1 BOSONS}

We consider the mixture of ultracold spin- $1{ }^{87} \mathrm{Rb}$ and ${ }^{23} \mathrm{Na}$ atoms at low magnetic field. In this system the inter-atomic interaction seriously depends on the atomic species. Explicitly, when the two atoms are of the same species $j$ ( $j=\mathrm{Rb}$ and $\mathrm{Na}$ for ${ }^{87} \mathrm{Rb}$ and ${ }^{23} \mathrm{Na}$, respectively) the interaction is given by $[1,2]$

$$
\hat{U}_{j}(\mathbf{r})=\left(\alpha_{j}+\beta_{j} \hat{\mathbf{F}}_{1} \cdot \hat{\mathbf{F}}_{2}\right) \delta(\mathbf{r}),
$$

when the two atoms are of different species the interaction is given by [24]

$$
\hat{U}_{\mathrm{Rb}-\mathrm{Na}}(\mathbf{r})=\left(\alpha+\beta \hat{\mathbf{F}}_{1} \cdot \hat{\mathbf{F}}_{2}+\gamma \hat{\mathcal{P}}_{0}\right) \delta(\mathbf{r}) .
$$

Here $\mathbf{r}$ is the inter-atomic relative coordinate, $\hat{\mathbf{F}}_{1}$ and $\hat{\mathbf{F}}_{2}$ are the respective spin operators of the two atoms, and $\hat{\mathcal{P}}_{0}$ is the projection operator for the two-body hyperfine state corresponding to total spin $\hat{\mathbf{F}}_{\text {tot }} \equiv \hat{\mathbf{F}}_{1}+\hat{\mathbf{F}}_{2}=0$. The interaction intensities $\left(\alpha_{j}, \beta_{j}, \alpha, \beta, \gamma\right)(j=\mathrm{Rb}, \mathrm{Na})$ are given by

$$
\begin{aligned}
& \alpha_{j}=\left(\frac{4 \pi \hbar^{2}}{3 M_{j}}\right)\left(a_{j}^{(0)}+2 a_{j}^{(2)}\right) ; \\
& \beta_{j}=\left(\frac{4 \pi \hbar^{2}}{3 M_{j}}\right)\left(a_{j}^{(2)}-a_{j}^{(0)}\right) ;
\end{aligned}
$$

and

$$
\begin{aligned}
& \alpha=\left(\frac{\pi \hbar^{2}}{\mu}\right)\left(a_{\mathrm{Rb}-\mathrm{Na}}^{(0)}+2 a_{\mathrm{Rb}-\mathrm{Na}}^{(2)}\right) ; \\
& \beta=\left(\frac{\pi \hbar^{2}}{\mu}\right)\left(a_{\mathrm{Rb}-\mathrm{Na}}^{(2)}-a_{\mathrm{Rb}-\mathrm{Na}}^{(1)}\right) ; \\
& \gamma=\left(\frac{\pi \hbar^{2}}{\mu}\right)\left(2 a_{\mathrm{Rb}-\mathrm{Na}}^{(0)}-3 a_{\mathrm{Rb}-\mathrm{Na}}^{(1)}+a_{\mathrm{Rb}-\mathrm{Na}}^{(2)}\right) .
\end{aligned}
$$

Here $M_{j}$ and $a_{j}^{\left(F_{\mathrm{tot}}\right)}\left(j=\mathrm{Rb}, \mathrm{Na} ; F_{\mathrm{tot}}=0,2\right)$ are the mass and $s$-wave scattering length of a single atom of type $j$, respectively; while $\mu=\frac{M_{\mathrm{Rb}} M_{\mathrm{Na}}}{M_{\mathrm{Rb}}+M_{\mathrm{Na}}}$, and $a_{\mathrm{Rb}-\mathrm{Na}}^{\left(F_{\mathrm{tot}}\right)}\left(F_{\mathrm{tot}}=0,1,2\right)$ are the reduced mass and $s$ wave scattering length of two atoms of different species with total spin $F_{\text {tot }}$, respectively. Previous measurements show that $\left(\alpha_{\mathrm{Rb}}, \beta_{\mathrm{Rb}}\right)=\frac{4 \pi \hbar^{2} a_{0}}{M_{\mathrm{Rb}}}(100.9,-0.47)[2]$, $\left(\alpha_{\mathrm{Na}}, \beta_{\mathrm{Na}}\right)=\frac{4 \pi \hbar^{2} a_{0}}{M_{\mathrm{Na}}}(52.66,1.88)$ [37]. The theoretical calculations show that $(\alpha, \beta)=\frac{2 \pi \hbar^{2} a_{0}}{\mu}(78.9,-2.5)$ and $\gamma=(0.06) \frac{2 \pi \hbar^{2} a_{0}}{\mu}[24,38]$, with $a_{0}$ being the Bohr radius.

Since both ${ }^{87} \mathrm{Rb}$ and ${ }^{23} \mathrm{Na}$ atoms are considered in the $F=1$ hyperfine manifold, each atom has three hyperfine states corresponding to magnetic quantum number $m=$ $0, \pm 1$. The above two-body interactions can induce the 
following seven spin-changing scattering processes:

$$
\begin{array}{lll}
\langle 1\rangle \quad \mathrm{Rb}_{\mathrm{R}}: & \mathrm{Rb} \mathrm{b}_{-1}+\mathrm{Rb}_{+1} \rightleftharpoons \mathrm{Rb}_{0}+\mathrm{Rb}_{0} ; \\
\langle 2\rangle \quad \beta_{\mathrm{Na}}: & \mathrm{Na} \mathrm{Na}_{-1}+\mathrm{Na}_{+1} \rightleftharpoons \mathrm{Na}_{0}+\mathrm{Na}_{0} ; \\
\langle 3\rangle \beta-\gamma / 3: & \mathrm{Rb}_{-1}+\mathrm{Na}_{+1} \rightleftharpoons \mathrm{Rb}_{0}+\mathrm{Na}_{0} ; \\
\langle 4\rangle \beta-\gamma / 3: & \mathrm{Rb}_{+1}+\mathrm{Na}_{-1} \rightleftharpoons \mathrm{Rb}_{0}+\mathrm{Na}_{0} ; \\
\langle 5\rangle \quad \beta: \quad \mathrm{Rb}_{0}+\mathrm{Na}_{+1} \rightleftharpoons \mathrm{Rb}_{+1}+\mathrm{Na}_{0} ; \\
\langle 6\rangle \quad \beta: \quad \mathrm{Rb}_{0}+\mathrm{Na}_{-1} \rightleftharpoons \mathrm{Rb}_{-1}+\mathrm{Na}_{0} ; \\
\langle 7\rangle \quad \gamma / 3: \quad \mathrm{Rb}-1+\mathrm{Na}_{+1} \rightleftharpoons \mathrm{Rb}_{+1}+\mathrm{Na}_{-1} \\
& \text { (singlet pairing). }
\end{array}
$$

In Eq. (8) we also show the corresponding interaction intensity before each reaction equation, and the subscripts of $\pm 1,0$ denote the magnetic quantum number $m$ of each atom. For instance, in the process $\langle 1\rangle$ the magnetic quantum numbers $\left(m_{1}, m_{2}\right)$ of the two ${ }^{87} \mathrm{Rb}$ atoms can be changed from $(-1,+1)$ to $(0,0)$ and vice versa. The processes $\langle 1,2\rangle$ and $\langle 3\rangle-\langle 7\rangle$ are intra-species and interspecies spin-changing collisions, respectively.

The above process $\langle 7\rangle$ is the singlet-pairing process [26], as mentioned above. In this process the magnetic quantum number of each atom can be changed by \pm 2 , while in all other processes $\langle 1\rangle-\langle 6\rangle$ the single-atom magnetic quantum number can only be changed by \pm 1 . In the following section we show our approach for the laser control of this process.

\section{CONTROL OF SINGLET-PAIRING PROCESS VIA LASER-INDUCED SMF}

In this work consider the cases with low magnetic field (less than four Gauss). Under this condition no magnetic Feshbach resonance [38, 39] for our system has been discovered, and thus the interaction intensities $\left(\alpha_{j}, \beta_{j}, \alpha, \beta, \gamma\right)(j=\mathrm{Rb}, \mathrm{Na})$ cannot be changed via the magnetic field. Nevertheless, one can still efficiently control the spin-changing processes by changing the detuning between the two-atom Zeeman-energies before and after each process. This detuning can be denoted as $\Delta_{\langle l\rangle}$ for the process $\langle l\rangle(l=1,2, \ldots, 7)$. For instance, for the singlet-pairing process $\langle 7\rangle$ we have

$$
\Delta_{\langle 7\rangle}=\left(E_{-1}^{(\mathrm{Rb})}+E_{+1}^{(\mathrm{Na})}\right)-\left(E_{+1}^{(\mathrm{Rb})}+E_{-1}^{(\mathrm{Na})}\right)
$$

with $E_{m}^{(j)}(j=\mathrm{Rb}, \mathrm{Na} ; m=0, \pm 1)$ being the free energy of a $j$-atom with magnetic quantum number $m$. For our weakly-interacting systems, the effect of the spinchanging process $\langle l\rangle(l=1,2, \ldots, 7)$ is usually significant when $\Delta_{\langle l\rangle}=0$, i.e., when the hypferfine states before and after the scattering are resonant with each other. Accordingly, the effect of process $\langle l\rangle$ is weak when the detuning $\Delta_{\langle l\rangle}$ is far away from zero.

Our purpose of this work is to enhance the effect of the singlet-pairing process $\langle 7\rangle$ and simultaneously suppress the effect of other processes. According to the above discussion, we can realize this by tuning the detuning $\Delta_{\langle 7\rangle}$ to zero while keeping the detunings for other processes to finite, i.e.,

$$
\begin{aligned}
& \Delta_{\langle 7\rangle}=0 \\
& \Delta_{\langle l\rangle} \neq 0 \text { for } l=1, \ldots, 6 .
\end{aligned}
$$

For realistic systems the above condition can be expressed more explicitly as

$$
\begin{aligned}
& \left|\frac{\Delta_{\langle 7\rangle}}{g_{\langle 7\rangle}}\right| \ll 1 ; \\
& \left|\frac{\Delta_{\langle l\rangle}}{g_{\langle l\rangle}}\right| \gtrsim 1, \text { for } l=1, \ldots, 6,
\end{aligned}
$$

where $g_{\langle l\rangle}(l=1, \ldots, 7)$ is the characteristic interaction intensity for the process $\langle l\rangle[40]$.

\section{A. The case only with the real magnetic field}

We first consider the case without laser-induced species-dependent SMF. In this case the free energies $E_{m}^{(j)}(j=\mathrm{Rb}, \mathrm{Na} ; m=0, \pm 1)$ as well as the detunings $\Delta_{\langle l\rangle}(l=1,2, \ldots, 7)$ can only be tuned via the real magnetic field $B \mathbf{e}_{z}$, with $\mathbf{e}_{z}$ being the unit vector along the $z$-direction. Explicitly, we have

$$
E_{m}^{(j)}=-p^{(j)} m B+q^{(j)} m^{2} B^{2} \quad(j=\mathrm{Rb}, \mathrm{Na}) .
$$

Here the first and second terms are the linear and quadratic Zeeman effects, respectively, with $p^{(j)}$ and $q^{(j)}$ being the corresponding coefficients. Explicitly, we have $\left(p^{(\mathrm{Rb})}, p^{(\mathrm{Na})}\right) / h=(702369,702023)(\mathrm{Hz} / \mathrm{G})$ and $\left(q^{(\mathrm{Rb})}, q^{(\mathrm{Na})}\right) / h=(72,277)\left(\mathrm{Hz} / \mathrm{G}^{2}\right)[41,42]$.

According to Eq. (14) and Eq. (9), the detuning $\Delta_{\langle 7\rangle}$ for the singlet-pairing process $\langle 7\rangle$ is

$$
\Delta_{\langle 7\rangle}=2\left(p^{(\mathrm{Rb})}-p^{(\mathrm{Na})}\right) B .
$$

Since $p^{(\mathrm{Rb})} \neq p^{(\mathrm{Na})}$, Eq. (15) yields that $\Delta_{\langle 7\rangle}$ is zero only when $B=0$. However, according to Eq. (14), in this case the detunings $\Delta_{\langle 1, \ldots, 6\rangle}$ of other spin-changing processes are all zero. Therefore, the conditions (10) and (11) cannot be simultaneously satisfied with only a real magnetic field and no synthetic field.

\section{B. Our proposal}

Now we show our proposal for the control of the singlet pairing process. We assume that in our system there is not only the weak real magnetic field $B \mathbf{e}_{z}$ but also a laser beam with $\sigma_{+^{-}}$or $\sigma_{-}$-polarization, which is far off resonant for the D1 and D2 transitions of atom of both ${ }^{87} \mathrm{Rb}$ atom and ${ }^{23} \mathrm{Na}$ atom. As shown in Fig. 1, this beam can induce an AC-Stark energy shift $\delta E_{m}^{(j)}$ for the hyperfine state of a $j$-atom $(j=\mathrm{Rb}, \mathrm{Na})$ with magnetic quantum number $m(m=0, \pm 1)$. Here we 

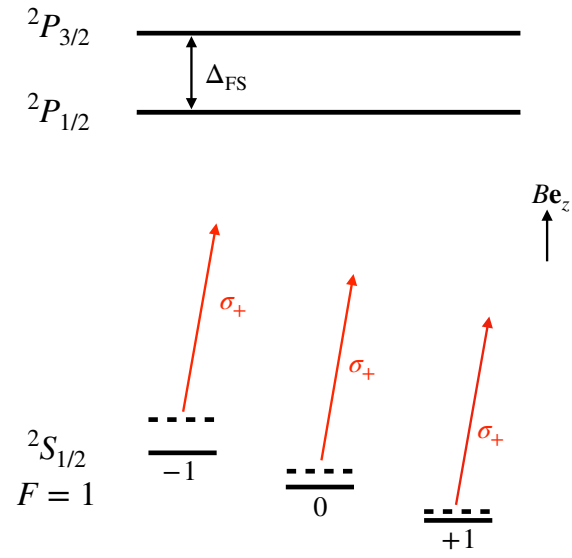

FIG. 1. (Color online) Schematic diagram for the laserinduced SMF for a spin-1 ultracold alkaline atom, i.e., the result in Eq. (16). The solid black lines are the Zeeman levels of the ${ }^{2} \mathrm{~S}_{1 / 2}$ states with $F=1$ for the case without the laser beam, which is totally determined by the real magnetic field $B \mathbf{e}_{z}$. The dashed black lines are the levels with both the real magnetic field and a $\sigma_{+}$-polarized laser beam. $\Delta_{\mathrm{FS}}$ is the fine-splitting of the ${ }^{2} \mathrm{P}_{3 / 2}$ and ${ }^{2} \mathrm{P}_{1 / 2}$ levels.

emphasis that the value of $\delta E_{m}^{(j)}$ depends on both the atomic species $j$ and magnetic quantum number $m$. The dependence of $\delta E_{m}^{(j)}$ on $m$ is essentially due to the fine splitting bewteen the D1 and D2 transitions, i.e., the energy gap $\Delta_{\mathrm{FS}}$ between the ${ }^{2} \mathrm{P}_{1 / 2}$ and ${ }^{2} \mathrm{P}_{3 / 2}$ states of an alkaline atom [36]. When the detunings of the laser beam for the D1 and D2 transitions are much larger than $\Delta_{\mathrm{FS}}$, for our system $\delta E_{m}^{(j)}(j=\mathrm{Rb}, \mathrm{Na})$ can be expressed as

$$
\begin{aligned}
& \delta E_{m}^{(j)}=S^{(j)} I-\chi V^{(j)} m I, \\
& \left(\chi= \pm 1 \text { for laser beam with } \sigma_{ \pm} \text {polarization }\right)
\end{aligned}
$$

with $I$ being the laser intensity. In the right-hand-side of Eq. (16) the $m$-independent term and the linear term of $m$ are called as scalar and vector light shifts, respectively. The coefficients $S^{(j)}$ and $V^{(j)}(j=\mathrm{Rb}, \mathrm{Na})$ are determined by the laser frequency and the electronic dipoletransition matrix element of the $j$-atom. If the laser beam has $\sigma_{-}$-polarization, the result is quite similar. The derivation of Eq. (16) and the general introduction for the vector light shift can be found in the review article [36] and the references therein.

Combining Eq. (16) and Eq. (14), we can obtain the energy of a $j$-atom $(j=\mathrm{Rb}, \mathrm{Na})$ with magnetic quantum number $m(m=0, \pm 1)$ in the presence of both real magnetic field $B$ and laser-induced vector light shift:

$$
\begin{array}{r}
E_{m}^{(j)}=S^{(j)} I-p^{(j)} m\left(B+B_{\mathrm{L}}^{(j)}\right)+q^{(j)} m^{2} B^{2}, \\
(j=\mathrm{Rb}, \mathrm{Na}),
\end{array}
$$

where the factor $B_{\mathrm{L}}^{(j)}$ is defined as

$$
B_{\mathrm{L}}^{(j)}=\chi \frac{V^{(j)}}{p^{(j)}} I
$$

and describes the contribution from the vector light shift. It is clear that the effect of the vector light shift is same as the linear Zeeman shift given by a synthetic magnetic field (SMF) $B_{\mathrm{L}}^{(j)} \mathbf{e}_{z}(j=\mathrm{Na}, \mathrm{Rb})$.

Eqs. (17) and (9) yield that in the presence of the the laser beam, the detuning $\Delta_{\langle 7\rangle}$ for the singlet pairing process becomes

$$
\begin{aligned}
\Delta_{\langle 7\rangle}= & 2\left(p^{(\mathrm{Rb})}-p^{(\mathrm{Na})}\right) B \\
& +2\left(p^{(\mathrm{Rb})} B_{\mathrm{L}}^{(\mathrm{Rb})}-p^{(\mathrm{Na})} B_{\mathrm{L}}^{(\mathrm{Na})}\right) .
\end{aligned}
$$

Therefore, the resonance condition $\Delta_{\langle 7\rangle}=0$ for the singlet pairing process can be satisfied under a finite real magnetic field, i.e., when

$$
B=B_{0} \equiv-\frac{1}{2}\left(B_{\mathrm{L}}^{(\mathrm{Rb})}+B_{\mathrm{L}}^{(\mathrm{Na})}\right)+R\left(B_{\mathrm{L}}^{(\mathrm{Na})}-B_{\mathrm{L}}^{(\mathrm{Rb})}\right)
$$

with the ratio $R$ being defined as

$$
R=\frac{p^{(\mathrm{Rb})}+p^{(\mathrm{Na})}}{2\left(p^{(\mathrm{Rb})}-p^{(\mathrm{Na})}\right)}
$$

As shown above, the values of $p^{(\mathrm{Rb})}$ and $p^{(\mathrm{Na})}$ are very close to each other. Due to this fact, the ratio $R$ in Eq. (20) is very large:

$$
R \approx 2.03 \times 10^{3}
$$

In the following discussions, for simplicity, we assume that $B_{\mathrm{L}}^{(\mathrm{Na})}$ is negligibly small, while $B_{\mathrm{L}}^{(\mathrm{Rb})}$ is much larger than $B_{\mathrm{L}}^{(\mathrm{Na})}$. This is easy to be realized because ${ }^{87} \mathrm{Rb}$ and ${ }^{23} \mathrm{Na}$ atoms have very different electronic structures [24]. In this case we can only take into account $B_{\mathrm{L}}^{(\mathrm{Rb})}$ in our calculations. Thus, according to Eqs. (20) and (22), the resonance point $B_{0}$ for the singlet pairing process is

$$
B_{0} \approx-\left(\frac{1}{2}+R\right) B_{\mathrm{L}}^{(\mathrm{Rb})} \approx-2030 \times B_{\mathrm{L}}^{(\mathrm{Rb})} .
$$

In realistic experiments, the laser-induced $\mathrm{SMF} B_{\mathrm{L}}^{(\mathrm{Rb})}$ is usually of the order of milli Gauss (mG) or even weaker, so that the laser-induced heating effect is not too strong. Nevertheless, Eq. (23) shows that in the presence of this weak SMF we can realize the resonance condition $\Delta_{\langle 7\rangle}=0$ under a real magnetic field $B_{0}$ which is as large as several Gauss. On the other hand, at such a large real magnetic field, the detuning $\Delta_{\langle 1, \ldots, 6\rangle}$ for other spinchanging processes can be large enough. Therefore, the conditions (10) and (11) can be satisfied simultaneously. This is the basic principle of our proposal.

We illustrate the above principle in Fig. 2. In Fig. 2(a) we show the variation of the detuning $\Delta_{\langle 7\rangle} / h$ with real magnetic field $B$, for the cases with different laserinduced synthetic magnetic field $B_{\mathrm{L}}^{(\mathrm{Rb})}$. It is shown that with the help of $B_{\mathrm{L}}^{(\mathrm{Rb})}$ we can realize $\Delta_{\langle 7\rangle}=0$ for $B \neq 0$. In Fig. 2(b) we illustrate the detunings $\Delta_{\langle l\rangle} / h$ 

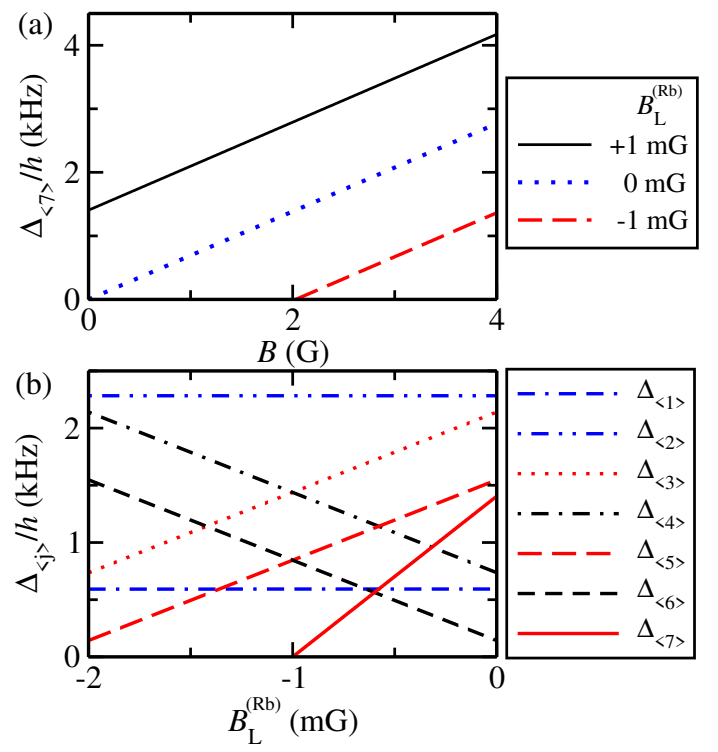

FIG. 2. (Color online) (a): The detuning $\Delta_{\langle 7\rangle}$ as a function of real magnetic field $B$, for the cases with laser-induced SMF $B_{\mathrm{L}}^{(\mathrm{Na})}=0$ and $B_{\mathrm{L}}^{(\mathrm{Rb})}=+1 \mathrm{mG}$ (black solid line), $B_{\mathrm{L}}^{(\mathrm{Rb})}=$ $0 \mathrm{mG}$ (blue dotted line), $B_{\mathrm{L}}^{(\mathrm{Rb})}=-1 \mathrm{mG}$ (red dashed line). (b): The detunings $\Delta_{\langle 1, \ldots 7\rangle}$ for all the seven spin-changing processes as a function of $B_{\mathrm{L}}^{(\mathrm{Rb})}$, for the cases with $B_{\mathrm{L}}^{(\mathrm{Na})}=0$ and the real magnetic field $B=2.03 \mathrm{G}$.

$(l=1, \ldots, 7)$ of all spin-changing scattering processes as functions of the laser-induced magnetic field $B_{\mathrm{L}}^{(\mathrm{Rb})}$, for the case with real magnetic field $B=2.03 \mathrm{G}$. It is clearly shown that by changing $B_{\mathrm{L}}^{(\mathrm{Rb})}$ one can tune $\Delta_{\langle 7\rangle}$ to be zero while keep the detunings $\Delta_{\langle 1, \ldots 6\rangle} / h$ of other processes to be as large as several $\mathrm{kHz}$.

\section{SPIN OSCILLATION OF TWO TRAPPED ATOMS}

In the above section we show our approach for the control of singlet-pairing process via a light-induced SMF. Now we apply this approach on a simple system with one ${ }^{87} \mathrm{Rb}$ and one ${ }^{23} \mathrm{Na}$ atom. We assume these two atoms are confined in an isotropic harmonic trap, e.g., an optical tweezer or a site of an optical lattice. For simplicity, in this section we further assume the confinement has the same angular frequency $\omega$ for each atom[43, 44]. Thus, the center-of-mass motion is decoupled with the relative motion, and in our calculation we can only consider the quantum state of two-body relative motion and spin. The Hilbert space $\mathcal{H}$ of our system is given by $\mathcal{H}=\mathcal{H}_{r} \otimes \mathcal{H}_{s \mathrm{Rb}} \otimes \mathcal{H}_{s \mathrm{Na}}$, with $\mathcal{H}_{r}$ being the Hilbert space for the two-atom spatial relative motion, and $\mathcal{H}_{s j}$ ( $j=\mathrm{Rb}, \mathrm{Na}$ ) being the one for the internal state of the $j$-atom. Here we use the symbol |\rangle$\rangle$ to denote the state in $\mathcal{H},|\rangle_{r}$ for state in $\mathcal{H}_{r}$, and $|m\rangle_{j}(j=\mathrm{Rb}, \mathrm{Na})$ for the state in $\mathcal{H}_{s j}$ with magnetic quantum number $m$.
As in Sec. III, in our calculation we only take into account the real magnetic field $B$ and the laser-induced $\mathrm{SMF} B_{\mathrm{L}}^{(\mathrm{Rb})}$ for the ${ }^{87} \mathrm{Rb}$ atom, and assume the laserinduced SMF for the ${ }^{23} \mathrm{Na}$ is negligible. Accordingly, the Hamiltonian for our problem is

$$
\hat{H}=\hat{H}_{\mathrm{ho}}+\hat{U}_{\mathrm{Rb}-\mathrm{Na}}(\mathbf{r})+\hat{Z}\left(B, B_{\mathrm{L}}^{(\mathrm{Rb})}\right),
$$

with

$$
\hat{H}_{\mathrm{ho}}=\frac{\hat{\mathbf{p}}^{2}}{2 \mu}+\frac{\mu}{2} \omega^{2} \mathbf{r}^{2}
$$

where $\hat{\mathbf{p}}$ is the relative-momentum operator of the two atoms, $\mu$ and $\mathbf{r}$ are the reduced mass and relative position of these two atoms as before, and the inter-species interaction $\hat{U}_{\mathrm{Rb}-\mathrm{Na}}(\mathbf{r})$ is given by Eq. (2). Here the rindependent operator $\hat{Z}\left(B, B_{\mathrm{L}}^{(\mathrm{Rb})}\right)$ describes the influence of the real and synthetic magnetic field on the energy of atomic spin states, and can be expressed as

$$
\begin{aligned}
& \hat{Z}\left(B, B_{\mathrm{L}}^{(\mathrm{Rb})}\right) \\
= & \sum_{m, m^{\prime}}\left(E_{m}^{(\mathrm{Rb})}+E_{m^{\prime}}^{(\mathrm{Na})}\right)|m\rangle_{\mathrm{Rb}}\left\langle m|\otimes| m^{\prime}\right\rangle_{\mathrm{Na}}\left\langle m^{\prime}\right|,
\end{aligned}
$$

where both $m$ and $m^{\prime}$ can take value $(0, \pm 1)$ and the energy $E_{m}^{(j)}(j=\mathrm{Rb}, \mathrm{Na})$ is given by Eq. (17).

Furthermore, we assume the relative wave function of the two atoms is initially prepared in the ground state $|0\rangle_{r}$ of the Hamiltonian $\hat{H}_{\text {ho }}$ defined in Eq. (25). The interaction $\hat{U}_{\mathrm{Rb}-\mathrm{Na}}(\mathbf{r})$ can induce the transition between $|0\rangle_{r}$ and the excited states of $\hat{H}_{\text {ho }}$. Nevertheless, the direct calculation shows that these transitions can be neglected when the inter-atomic scattering length $a_{\mathrm{Rb}-\mathrm{Na}}^{(0,1,2)}$ is much smaller than the characteristic length

$$
a_{\mathrm{ho}} \equiv \sqrt{\frac{\hbar}{\mu \omega}}
$$

of the trap [45]. As shown above, the values of $a_{\mathrm{Rb}-\mathrm{Na}}^{(0,1,2)}$ are less than $100 a_{0}$, while in almost all of the realistic experiments the confinement characteristic length $a_{\text {ho }}$ is larger than $1000 a_{0}$. Therefore, in the lowest-order calculation we can neglect the transition between different spatial states, i.e., assume the relative motion of the atoms is frozen in the state $|0\rangle_{r}$.

In addition, the inter-atomic interaction $\hat{U}_{\mathrm{Rb}-\mathrm{Na}}(\mathbf{r})$ can also induce the transtion between different hypferfine-spin states via the spin-changing processes shown in Sec. II. Here we consider the system where the ${ }^{87} \mathrm{Rb}$ atom and ${ }^{23} \mathrm{Na}$ atom are prepared in hyperfine spin states $|-1\rangle_{\mathrm{Rb}}$ and $|+1\rangle_{\mathrm{Na}}$, respectively. In this case, only the processes $\langle 3\rangle,\langle 4\rangle$ and $\langle 7\rangle$ can occur during the evolution, as shown in Fig. 3(a). As a result, the state at time $t$ can be expressed as

$$
|\Psi(t)\rangle\rangle=\sum_{\left\{m, m^{\prime}\right\}} C_{m, m^{\prime}}(t)|0\rangle_{r} \otimes|m\rangle_{\mathrm{Rb}} \otimes\left|m^{\prime}\right\rangle_{\mathrm{Na}}
$$


(a)

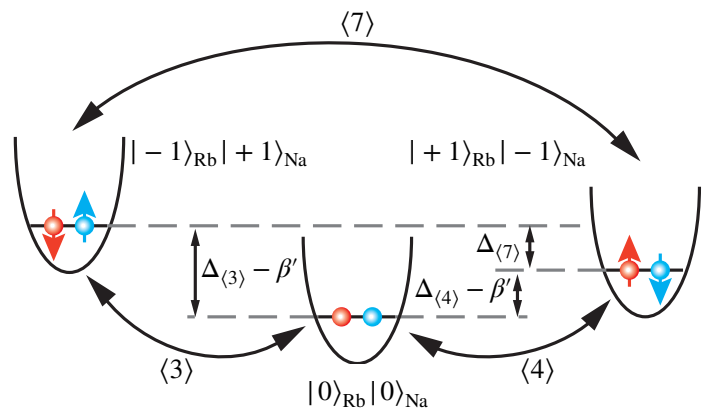

(b)

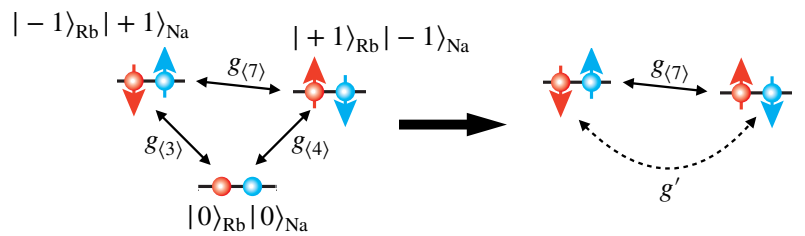

FIG. 3. (Color online) (a): A schematic illustration for the spin dynamics of a ${ }^{87} \mathrm{Rb}$ atom (red ball) and a ${ }^{23} \mathrm{Na}$ atom (blue ball) confined in an isotropic Harmonic trap. Here we also show the effective detunings $\Delta_{\langle 3,4\rangle}-\beta^{\prime}$ for the processes $\langle 3,4\rangle$ and $\Delta_{\langle 7\rangle}$ for the process $\langle 7\rangle$ (see Sec. IV). (b): Under the condition (36) the state $|0\rangle_{\mathrm{Rb}}|0\rangle_{\mathrm{Na}}$ can be adiabatically eliminated. In this case the states $|-1\rangle_{\mathrm{Rb}}|+1\rangle_{\mathrm{Na}}$ and $\mid+$ $1\rangle_{\mathrm{Rb}}|-1\rangle_{\mathrm{Na}}$ experiences both the direct coupling $g_{\langle 7\rangle}$ and an effective coupling $g^{\prime}$ which is given by the virtual transitions between these two states and $|0\rangle_{\mathrm{Rb}}|0\rangle_{\mathrm{Na}}$ (i.e., the off-resonant processes $\langle 3,4\rangle)$.

where $\left\{m, m^{\prime}\right\}$ can take the values $\{-1,+1\},\{0,0\}$ and $\{+1,-1\}$. Furthermore, by projecting the Schrödinger equation in the subspace spanned by the three states $|0\rangle_{r} \otimes|m\rangle_{\mathrm{Rb}} \otimes\left|m^{\prime}\right\rangle_{\mathrm{Na}}$ involved in Eq. (28), we can obtain the equation for the coefficients $C_{m, m^{\prime}}(t)$ (up to a global phase factor):

$$
i \hbar \frac{d}{d t}\left(\begin{array}{c}
C_{-1,+1} \\
C_{0,0} \\
C_{+1,-1}
\end{array}\right)=\mathcal{M}\left(\begin{array}{c}
C_{-1,+1} \\
C_{0,0} \\
C_{+1,-1}
\end{array}\right),
$$

where the matrix $\mathcal{M}$ is given by

$$
\mathcal{M}=\left(\begin{array}{ccc}
-\beta^{\prime} & g_{\langle 3\rangle} & g_{\langle 7\rangle} \\
g_{\langle 3\rangle} & -\Delta_{\langle 3\rangle} & g_{\langle 4\rangle} \\
g_{\langle 7\rangle} & g_{\langle 4\rangle} & -\beta^{\prime}-\Delta_{\langle 7\rangle}
\end{array}\right),
$$

with the detunings $\Delta_{\langle 3,7\rangle}$ being defined in Sec. III. In Eq. (30) the characteristic interaction strength (effective spin-changing intensity) $g_{\langle 3,4,7\rangle}$ are given by the projection of the inter-atomic interaction $\hat{U}_{\mathrm{Rb}-\mathrm{Na}}(\mathbf{r})$ on the ground state $|0\rangle_{r}$ of the relative spatial motion, and can be expressed as

$$
\begin{aligned}
& g_{\langle 3\rangle}=g_{\langle 4\rangle}=\beta^{\prime}-\gamma^{\prime} / 3 ; \\
& g_{\langle 7\rangle}=\gamma^{\prime} / 3 .
\end{aligned}
$$

with

$$
\left(\alpha^{\prime}, \beta^{\prime}, \gamma^{\prime}\right)=\frac{1}{\pi^{3 / 2} a_{\mathrm{ho}}^{3}}(\alpha, \beta, \gamma)
$$

With the help of the above equations we can obtain a clear qualitative understanding for the spin dynamics of these two atoms. In our system each of the spin-changing process $\langle 3,4,7\rangle$ can induce a quantum transition between two spin states (Fig. 3), i.e.,

$$
\begin{array}{ll}
\langle 3\rangle \text { induces : } & |-1\rangle_{\mathrm{Rb}}|+1\rangle_{\mathrm{Na}} \leftrightarrow|0\rangle_{\mathrm{Rb}}|0\rangle_{\mathrm{Na}} ; \\
\langle 4\rangle \text { induces : } & |+1\rangle_{\mathrm{Rb}}|-1\rangle_{\mathrm{Na}} \leftrightarrow|0\rangle_{\mathrm{Rb}}|0\rangle_{\mathrm{Na}} ; \\
\langle 7\rangle \text { induces : } & |+1\rangle_{\mathrm{Rb}}|-1\rangle_{\mathrm{Na}} \leftrightarrow|-1\rangle_{\mathrm{Rb}}|+1\rangle_{\mathrm{Na}} .
\end{array}
$$

Furthermore, using Eq. (30) and the fact $\Delta_{\langle 4\rangle}=\Delta_{\langle 3\rangle}-$ $\Delta_{\langle 7\rangle}$, we find that the detuning $\delta_{\langle l\rangle}$ of the transition induced by the process $\langle l\rangle(l=3,4,7)$ is given by

$$
\delta_{\langle 3,4\rangle}=\Delta_{\langle 3,4\rangle}-\beta^{\prime}
$$

and

$$
\delta_{\langle 7\rangle}=\Delta_{\langle 7\rangle},
$$

while the direct coupling intensity corresponding to this transition is just $g_{\langle l\rangle}$. Thus, this transition is significant when $\left|\delta_{\langle l\rangle}\right| \ll\left|g_{\langle l\rangle}\right|$, and becomes negligible when $\left|\delta_{\langle l\rangle}\right| \gg$ $\left|g_{\langle l\rangle}\right|$. As shown in Sec. III, this can be realized with the help of the light-induced SMF $B_{\mathrm{L}}^{(\mathrm{Rb})}$ via our approach.

According to the above discussion, to enhance the singlet-pairing process $\langle 7\rangle$ and simultaneously suppress the direct effect of the other two processes, one requires to make

$$
\left|\delta_{\langle 7\rangle}\right| \ll\left|g_{\langle 7\rangle}\right| ; \quad\left|\delta_{\langle 3,4\rangle}\right| \gg\left|g_{\langle 3,4\rangle}\right| .
$$

Explicitly, under this condition the state $|0\rangle_{\mathrm{Rb}}|0\rangle_{\mathrm{Na}}$ can be adiabatically eliminated and the coefficients $C_{-1,+1}(t)$ and $C_{1,-1}(t)$ satisfy the effective Schrödinger equation (up to a constant)

$i \hbar \frac{d}{d t}\left(\begin{array}{l}C_{-1,+1} \\ C_{+1,-1}\end{array}\right)=\left(\begin{array}{cc}\delta_{\langle 7\rangle}, & g_{\langle 7\rangle}+g^{\prime} \\ g_{\langle 7\rangle}+g^{\prime} & 0\end{array}\right)\left(\begin{array}{l}C_{-1,+1} \\ C_{+1,-1}\end{array}\right)$,

with

$$
g^{\prime}=-\frac{g_{\langle 3\rangle}^{2}}{\delta_{\langle 3\rangle}}
$$

Here we have used the facts $g_{\langle 3\rangle}=g_{\langle 4\rangle}$ and $\delta_{\langle 3\rangle} \approx \delta_{\langle 4\rangle}$. Eq. (37) shows that in addition to the direct coupling $g_{\langle 7\rangle}$ induced by the process $\langle 7\rangle$, the states $|-1\rangle_{\mathrm{Rb}}|+1\rangle_{\mathrm{Na}}$ and $|+1\rangle_{\mathrm{Rb}}|-1\rangle_{\mathrm{Na}}$ also experiences an effective coupling $g^{\prime}$. This term is given by the virtual transitions between these two states and the far-off resonant state $|0\rangle_{\mathrm{Rb}}|0\rangle_{\mathrm{Na}}$, and is actually an indirect effect of the processes $\langle 3,4\rangle$ (Fig. 3(b)). When $\left|g_{\langle 7\rangle}\right|$ is comparable with or much larger than $\left|g^{\prime}\right|$, the spin-changing process $\langle 7\rangle$ makes considerable contribution for the the Rabi oscillation between $|-1\rangle_{\mathrm{Rb}}|+1\rangle_{\mathrm{Na}}$ and $|+1\rangle_{\mathrm{Rb}}|-1\rangle_{\mathrm{Na}}$. Therefore, one can observe the effect of the process $\langle 7\rangle$ and measure the corresponding interaction parameter $\gamma$ with the help of this Rabi oscillation.

As an example, we consider the case with trapping frequency $\omega=2 \pi \times 40 \mathrm{kHz}$ and a real magnetic field 

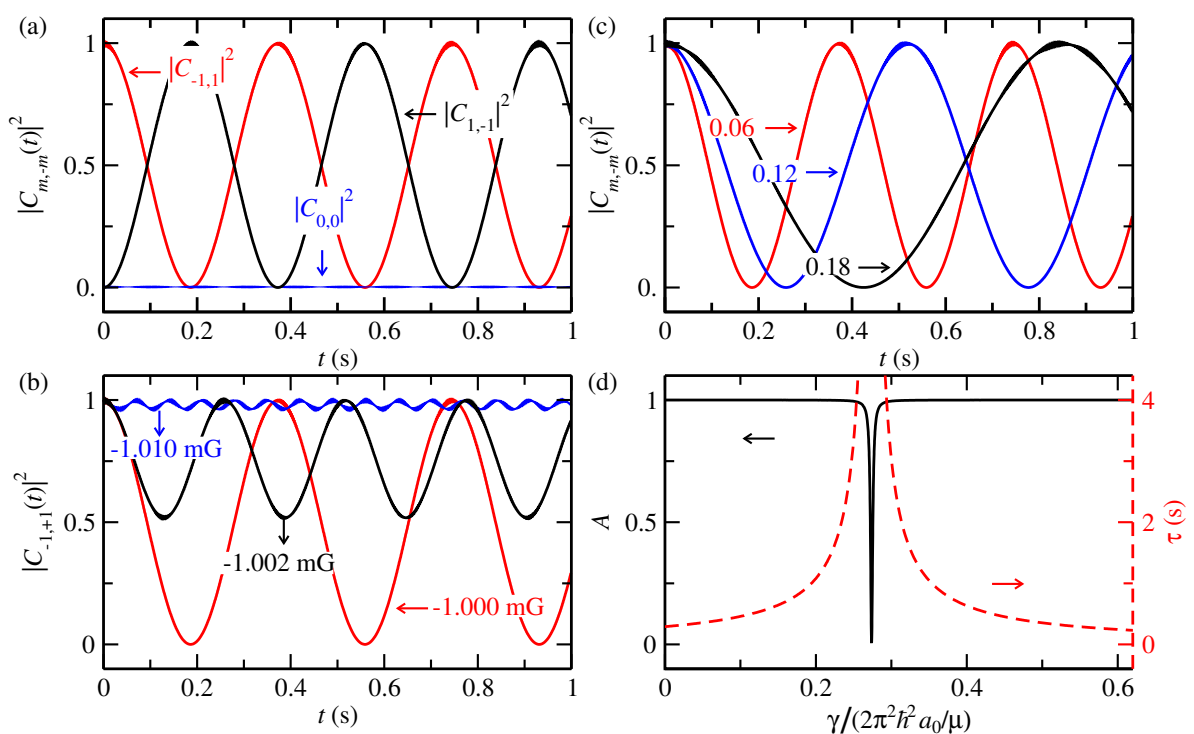

FIG. 4. (Color online) (a): $\left|C_{m, m^{\prime}}(t)\right|^{2}\left(m, m^{\prime}=0, \pm 1\right)$ given by the solution of Eq. (29) for initial spin state $|-1\rangle_{\mathrm{Rb}}|+1\rangle_{\mathrm{Na}}$. Here we consider the system with $\omega=2 \pi \times 40 \mathrm{kHz},(\alpha, \beta, \gamma)=\frac{2 \pi \hbar^{2} a_{0}}{\mu}(78.9,-2.5,0.06), B=2.03 \mathrm{G}, B_{\mathrm{L}}^{(\mathrm{Rb})}=-1 \mathrm{mG}$ and $B_{\mathrm{L}}^{(\mathrm{Na})}=0$. (b): $\left|C_{-1,+1}(t)\right|^{2}$ for the system of (a), with $B_{\mathrm{L}}^{(\mathrm{Rb})}=-1 \mathrm{mG}$ (red line), $-1.002 \mathrm{mG}$ (black line), $-1.010 \mathrm{mG}$ (blue line). (c): $\left|C_{-1,+1}(t)\right|^{2}$ for the system of (a), with $\gamma=(0.06) \frac{2 \pi \hbar^{2} a_{0}}{\mu}$ (red line), (0.12) $\frac{2 \pi \hbar^{2} a_{0}}{\mu}$ (blue line), (0.18) $\frac{2 \pi \hbar^{2} a_{0}}{\mu}$ (black line). (d): The amplitude $A$ and period $\tau$ of the time oscillation of the profile of $\left|C_{-1,+1}(t)\right|^{2}$ as a function of the interaction parameter $\gamma$. Other parameters are same as (a).

$B=2.03 \mathrm{G}$. According to the calculation in the above section, for such a system when the laser-induced SMF is tuned to $B_{\mathrm{L}}^{(\mathrm{Rb})}=-1 \mathrm{mG}$, we have $\delta_{\langle 7\rangle} / h=0.022 \mathrm{~Hz}=$ $0.054 g_{\langle 7\rangle} / h$ and $\left|\delta_{\langle 3,4\rangle}\right|=29\left|g_{\langle 3,4\rangle}\right|$. Therefore, in this case the condition (36) is satisfied and thus the Rabi oscillation $|-1\rangle_{\mathrm{Rb}}|+1\rangle_{\mathrm{Na}} \leftrightarrow|+1\rangle_{\mathrm{Rb}}|-1\rangle_{\mathrm{Na}}$ can be enhanced. To illustrate this effect, we exactly solve the three-level Schrödinger equation (29) for the initial spin state $|-1\rangle_{\mathrm{Rb}}|+1\rangle_{\mathrm{Na}}$, and show the time-evolution of the populations for each spin state in Fig. 4(a). It is clearly shown that the amplitudes of this Rabi oscillation (i.e., the amplitudes of the time oscillations of $\left|C_{-1,+1}(t)\right|^{2}$ and $\left.\left|C_{+1,-1}(t)\right|^{2}\right)$ is almost unit, while the population of the state $|0\rangle_{\mathrm{Rb}}|0\rangle_{\mathrm{Na}}$ is almost zero. In Fig. 4(b), we show the time oscillation of the population $\left|C_{-1,1}(t)\right|^{2}$ for various other values of the $\operatorname{SMF} B_{\mathrm{L}}^{(\mathrm{Rb})}$. It is clearly shown that both the period and amplitude of the oscillations are extremely sensitive to $B_{\mathrm{L}}^{(\mathrm{Rb})}$.

Our further calculation shows that in this system we have $g^{\prime}=-4.3 g_{\langle 7\rangle}$. Therefore, the singlet-pairing process $\langle 7\rangle$ makes considerable and observable contribution for the above Rabi oscillation, although it is still less than the contribution from the indirect coupling $g^{\prime}$ because of the extremely-weak interaction parameter $\gamma$ of the ${ }^{23} \mathrm{Na}-$ ${ }^{87} \mathrm{Rb}$ mixture $\left(\gamma \approx 0.024 \beta=(0.06) \frac{2 \pi \hbar^{2} a_{0}}{\mu}\right.$, as shown in Sec. II). in Fig. 4(c), we show the time oscillation of the population $\left|C_{-1,1}(t)\right|^{2}$ for various other values of $\gamma$, which are near the above realistic one. It is clearly shown that the period $\tau$ of this oscillation seriously depends on $\gamma$. Therefore, one can precisely measure the value of $\gamma$ by detecting $\tau$. In Fig. 4(d), we further illustrate the period $\tau$ and the amplitude $A$ of the Rabi oscillation $|-1\rangle_{\mathrm{Rb}} \mid+$ $1\rangle_{\mathrm{Na}} \leftrightarrow|+1\rangle_{\mathrm{Rb}}|-1\rangle_{\mathrm{Na}}$ as functions of $\gamma$, respectively. It is shown that if $\gamma$ were taking a particular value $\gamma_{*} \equiv$ (0.26) $\frac{2 \pi \hbar^{2} a_{0}}{\mu}$, we would have $\tau=\infty$ and $A=0$, i.e., this Rabi oscillation would be totally suppressed. That is just because for our system the coupling parameters $g_{\langle 7\rangle}$ and $g^{\prime}$ satisfy $g_{\langle 7\rangle}+g^{\prime}=0$ for $\gamma=\gamma_{*}$. Namely, there is a completely destructive interference between the direct and indirect transitions from $|+1\rangle_{\mathrm{Rb}}|-1\rangle_{\mathrm{Na}}$ to $|-1\rangle_{\mathrm{Rb}}|+1\rangle_{\mathrm{Na}}$, which are induced by the singlet-pairing process $\langle 7\rangle$ and the virtual processes through $|0\rangle_{\mathrm{Rb}}|0\rangle_{\mathrm{Na}}$, respectively. In the region around $\gamma=\gamma_{*}$, the period $\tau$ and the amplitude $A$ sensitively dependent on the value of $\gamma$, and thus can be used for the precise measurement this interaction parameter [46].

In the end of this section, we emphasis that the systems of two ultracold atoms confined in a single trap have been prepared in many experiments with optical lattice site or optical tweezer [47-49], and has been used for the inter-atomic interaction intensity for alkaline-earth (like) atoms [50, 51]. Therefore, the spinoscillation processes studied in this section are also possibly to be realized in current experiments, with which one can observe the singlet-pairing and measure the 
corresponding interaction intensity $\gamma$, as shown above. In addition, using these processes one can also prepare the two-atom entangled states, e.g., the Bell state $\left(|-1\rangle_{\mathrm{Na}}|+1\rangle_{\mathrm{Rb}} \pm i|+1\rangle_{\mathrm{Na}}|-1\rangle_{\mathrm{Rb}}\right) / \sqrt{2}$ which can be used for for the studies of quantum information or quantum physics.

\section{BINARY MIXTURES OF SPIN-1 BECS}

Now we study the control of the singlet-pairing process in a two-species BEC of spin- $1{ }^{87} \mathrm{Rb}$ and ${ }^{23} \mathrm{Na}$ atoms with our approach. As in the above sections, we assume our system is confined in an isotropic harmonic trap, and there are both a real magnetic field and a laser-induced SMF. As shown in Appendix A, under the mean-field and single-mode approximations [1, 2, 52-57], the states of ${ }^{87} \mathrm{Rb}$ and ${ }^{23} \mathrm{Na}$ BECs can be described by three-component wave functions

$$
\psi(\mathbf{r})\left(\begin{array}{l}
\xi_{-1}(t), \\
\xi_{0}(t), \\
\xi_{+1}(t)
\end{array}\right) \quad \text { and } \quad \phi(\mathbf{r})\left(\begin{array}{c}
\zeta_{-1}(t), \\
\zeta_{0}(t), \\
\zeta_{+1}(t)
\end{array}\right)
$$

respectively. The spatial wave functions $\psi(\mathbf{r})$ and $\phi(\mathbf{r})$, which are normalized to unit, are determined by the Gross-Pitaevskii equations (A7, A8). In addition, the spin states of the ${ }^{87} \mathrm{Rb}$ and ${ }^{23} \mathrm{Na}$ BECs are described by the time-dependent complex row vectors $\left[\xi_{-1}(t), \xi_{0}(t), \xi_{+1}(t)\right]^{\mathrm{T}}$ and $\left[\zeta_{-1}(t), \zeta_{0}(t), \zeta_{+1}(t)\right]^{\mathrm{T}}$, respectively, which satisfy the normalization condition

$$
\sum_{m=0, \pm 1}\left|\xi_{m}\right|^{2}=\sum_{m=0, \pm 1}\left|\zeta_{m}\right|^{2}=1
$$

The time evolution of the components $\xi_{m}(t)$ and $\zeta_{m}(t)$ $(m=0, \pm 1)$, i.e., the spin dynamics of the ${ }^{87} \mathrm{Rb}$ and ${ }^{23} \mathrm{Na}$ BECs, are determined by the equations (Appendix A):

$$
\begin{aligned}
\imath \hbar \dot{\xi}_{ \pm 1}= & E_{ \pm 1}^{(\mathrm{Rb})} \xi_{ \pm 1}+\bar{\beta}_{\mathrm{Rb}}\left[\left(\left|\xi_{ \pm 1}\right|^{2}+\left|\xi_{0}\right|^{2}-\left|\xi_{\mp 1}\right|^{2}\right) \xi_{ \pm 1}+\xi_{0}^{2} \xi_{\mp 1}^{*}\right] \\
& +\bar{\beta}^{(\mathrm{Na})}\left[\left(\left|\zeta_{ \pm 1}\right|^{2}-\left|\zeta_{\mp 1}\right|^{2}\right) \xi_{ \pm 1}+\zeta_{ \pm 1} \xi_{0} \zeta_{0}^{*}+\zeta_{0} \xi_{0} \zeta_{\mp 1}^{*}\right]+\frac{\bar{\gamma}^{(\mathrm{Na})}}{3}\left(\zeta_{\mp 1} \xi_{ \pm 1}+\zeta_{ \pm 1} \xi_{\mp 1}-\zeta_{0} \xi_{0}\right) \zeta_{\mp 1}^{*} ; \\
\imath \hbar \dot{\xi}_{0}= & E_{0}^{(\mathrm{Rb})} \xi_{0}+\bar{\beta}_{\mathrm{Rb}}\left[\left(\left|\xi_{+1}\right|^{2}+\left|\xi_{-1}\right|^{2}\right) \xi_{0}+2 \xi_{+1} \xi_{-1} \xi_{0}^{*}\right] \\
& +\bar{\beta}^{(\mathrm{Na})}\left[\zeta_{0} \xi_{+1} \zeta_{+1}^{*}+\zeta_{0} \xi_{-1} \zeta_{-1}^{*}+\left(\zeta_{-1} \xi_{+1}+\xi_{-1} \zeta_{+1}\right) \zeta_{0}^{*}\right]+\frac{\bar{\gamma}^{(\mathrm{Na})}}{3}\left(-\zeta_{-1} \xi_{+1}+\zeta_{0} \xi_{0}-\zeta_{+1} \xi_{-1}\right) \zeta_{0}^{*} ; \\
\imath \hbar \dot{\zeta}_{ \pm 1}= & E_{ \pm 1}^{(\mathrm{Na})} \zeta_{ \pm 1}+\bar{\beta}_{\mathrm{Na}}\left[\left(\left|\zeta_{ \pm 1}\right|^{2}+\left|\zeta_{0}\right|^{2}-\left|\zeta_{\mp 1}\right|^{2}\right) \zeta_{ \pm 1}+\zeta_{0}^{2} \zeta_{\mp 1}^{*}\right] \\
& +\bar{\beta}^{(\mathrm{Rb})}\left[\left(\left|\xi_{ \pm 1}\right|^{2}-\left|\xi_{\mp 1}\right|^{2}\right) \zeta_{ \pm 1}+\xi_{ \pm 1} \zeta_{0} \xi_{0}^{*}+\xi_{0} \zeta_{0} \xi_{\mp 1}^{*}\right]+\frac{\bar{\gamma}^{(\mathrm{Rb})}}{3}\left(\zeta_{\mp 1} \xi_{ \pm 1}+\xi_{\mp 1} \zeta_{ \pm 1}-\zeta_{0} \xi_{0}\right) \xi_{\mp 1}^{*} ; \\
\imath \hbar \dot{\zeta}_{0}= & E_{0}^{(\mathrm{Na})} \zeta_{0}+\bar{\beta}_{\mathrm{Na}}\left[\left(\left|\zeta_{+1}\right|^{2}+\left|\zeta_{-1}\right|^{2}\right) \zeta_{0}+2 \zeta_{+1} \zeta_{-1} \zeta_{0}^{*}\right] \\
& +\bar{\beta}^{(\mathrm{Rb})}\left[\xi_{0} \zeta_{+1} \xi_{+1}^{*}+\xi_{0} \zeta_{-1} \xi_{-1}^{*}+\left(\xi_{-1} \zeta_{+1}+\xi_{+1} \zeta_{-1}\right) \xi_{0}^{*}\right]+\frac{\bar{\gamma}^{(\mathrm{Rb})}}{3}\left(-\zeta_{-1} \xi_{+1}+\xi_{0} \zeta_{0}-\zeta_{+1} \xi_{-1}\right) \xi_{0}^{*},
\end{aligned}
$$

with the effective interaction strengths being defined as

$$
\begin{aligned}
\bar{\beta}_{\mathrm{Rb}} & =\beta_{\mathrm{Rb}} N_{\mathrm{Rb}} \int d \mathbf{r}|\psi(\mathbf{r})|^{4}, \\
\bar{\beta}_{\mathrm{Na}} & =\beta_{\mathrm{Na}} N_{\mathrm{Na}} \int d \mathbf{r}|\phi(\mathbf{r})|^{4}, \\
\bar{\beta}^{(\mathrm{Rb}, \mathrm{Na})} & =\beta N_{\mathrm{Rb}, \mathrm{Na}} \int d \mathbf{r}|\psi(\mathbf{r})|^{2}|\phi(\mathbf{r})|^{2}, \\
\bar{\gamma}^{(\mathrm{Rb}, \mathrm{Na})} & =\gamma N_{\mathrm{Rb}, \mathrm{Na}} \int d \mathbf{r}|\psi(\mathbf{r})|^{2}|\phi(\mathbf{r})|^{2} .
\end{aligned}
$$

Here $N_{j}(j=\mathrm{Na}, \mathrm{Rb})$ are the number of the $j$-atoms. In Eqs. (41-44) the effective Zeeman energies $E_{0, \pm 1}^{(j)}(j=\mathrm{Na}$, $\mathrm{Rb})$ are functions of the real magnetic field $B$ and the laser-induced SMF $B_{\mathrm{L}}^{(\mathrm{Na}, \mathrm{Rb})}$, as defined in Eq. (17). Therefore, one can control these energies, and thus the detunings $\Delta_{\langle l\rangle}(l=1, \ldots, 7)$ for the spin-changing process, via $B$ and $B_{\mathrm{L}}^{(\mathrm{Na}, \mathrm{Rb})}$. As mentioned in Sec. I, we can enhance the singlet-pairing process $\langle 7\rangle$ by tuning $B$ and $B_{\mathrm{L}}^{(\mathrm{Na}, \mathrm{Rb})}$ to the proper values where this process is resonant while the other spin-changing processes are far-off resonant, i.e., the conditions $(10,11)$ are satisfied.

To illustrate the above technique, we investigate the spin dynamics for the case with $B=2.03 \mathrm{G}, B_{\mathrm{L}}^{(\mathrm{Rb})}=-1 \mathrm{mG}$ and $B_{\mathrm{L}}^{(\mathrm{Na})}$ is negligible, where the conditions $(10,11)$ can be satisfied. In our calculation we assume $\omega_{\mathrm{Rb}}=2 \pi \times 200$ $\mathrm{Hz}, \omega_{\mathrm{Na}}=2 \pi \times 500 \mathrm{~Hz}$, and $N_{\mathrm{Rb}}=N_{\mathrm{Na}}=4 \times 10^{4}$, and derive the atomic probability densities $|\psi(\mathbf{r})|^{2}$ and $|\phi(\mathbf{r})|^{2}$ via the Thomas-Fermi approximation (Appendix $\mathrm{B})$. As a result, the effective spin-singlet pairing interaction strength is $\bar{\gamma}^{(\mathrm{Rb}, \mathrm{Na})} / h=0.965 \mathrm{~Hz}$ and the corre- 

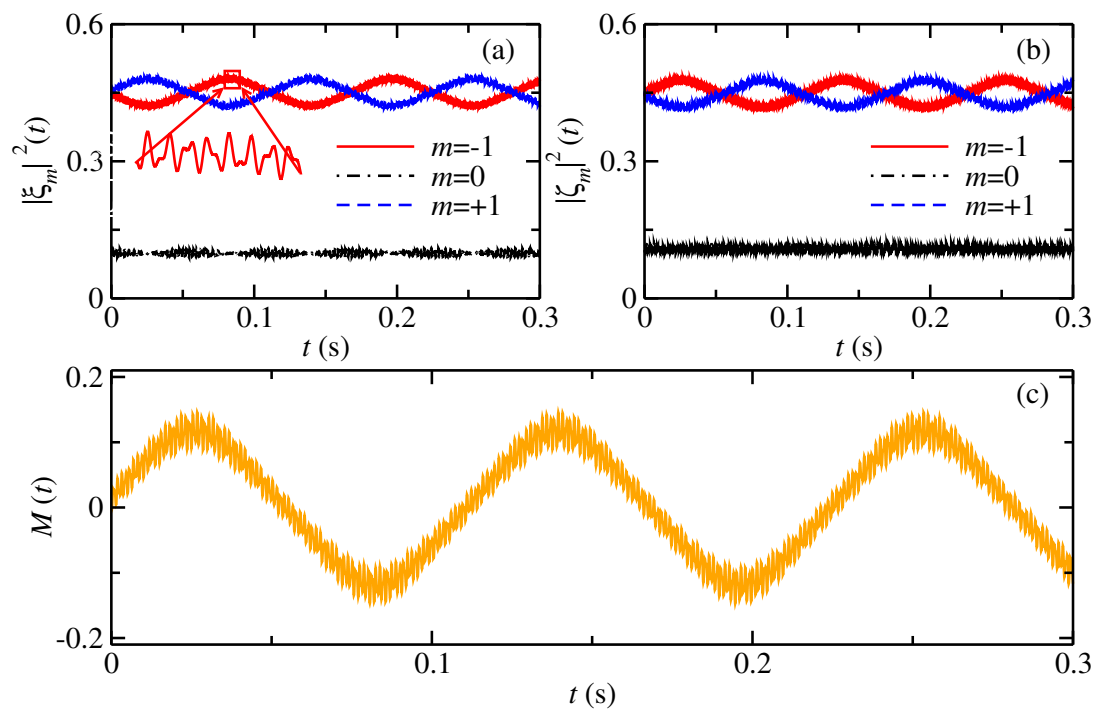

FIG. 5. (Color online) (a): The time evolution of the populations $\left|\xi_{m}(t)\right|^{2}$ and $\left|\zeta_{m}(t)\right|^{2}(m=0, \pm 1)$, which are given by the numerical solution of Eqs. (41-44) under the initial conditions (49,50). Other details of our calculation are shown in the main text of Sec. V. (b): The time evolution of the relative magnetization $M(t)$ for the system of (a).

sponding chemical potentials are $\mu_{\mathrm{Rb}} / h=3970 \mathrm{~Hz}$ and $\mu_{\mathrm{Na}} / h=6093 \mathrm{~Hz}$ (see Eqs. (A7-A8)). We numerically solve Eqs. (41)-(44) for this system, under the initial condition

$$
\begin{aligned}
& \left(\begin{array}{l}
\xi_{-1}(t=0) \\
\xi_{0}(t=0) \\
\xi_{+1}(t=0)
\end{array}\right)=\left(\begin{array}{l}
\sqrt{0.45} \\
\sqrt{0.10} \\
\sqrt{0.45}
\end{array}\right) ; \\
& \left(\begin{array}{l}
\zeta_{-1}(t=0) \\
\zeta_{0}(t=0) \\
\zeta_{+1}(t=0)
\end{array}\right)=\left(\begin{array}{l}
\sqrt{0.45} \imath \\
\sqrt{0.10} \\
\sqrt{0.45}
\end{array}\right) .
\end{aligned}
$$

Namely, $90 \%$ of the atoms are assumed to be initially prepared in the states with $m= \pm 1$. In Fig. $5(\mathrm{a}, \mathrm{b})$ we show the time evolution of the populations of ${ }^{87} \mathrm{Rb}$ and ${ }^{23} \mathrm{Na}$ atoms in each spin state, i.e., the functions $\left|\xi_{m}(t)\right|^{2}$ and $\left|\zeta_{m}(t)\right|^{2}(m=0, \pm 1)$. It is shown that each population rapidly oscillates with time with a small amplitude, around a slowly-varying central profile. The rapidlyoscillating details of these functions are essentially due to the nonlinearity of Eqs. (41)-(44). In the following we will only focus on the behaviors of the slowly-varying central profiles which give coarse-grained descriptions for the spin dynamics.

Fig. 5(a, b) clearly shows that for each type of atom, the central profiles of populations $\left|\xi_{0}(t)\right|^{2}$ and $\left|\zeta_{0}(t)\right|^{2}$ of the states with $m=0$ almost do not change with time. Thus the spin-changing processes $\langle 1, \ldots, 6\rangle$ defined in Eq. (8), in which the states with $m=0$ are involved, are all suppressed. On the other hand, Fig. $5(\mathrm{a}, \mathrm{b})$ also show that the central profiles of $\left|\xi_{ \pm 1}(t)\right|^{2}$ and $\left|\zeta_{ \pm 1}(t)\right|^{2}$ significantly oscillate with time. This behavior implies that the singlet-pairing process $\langle 7\rangle$ is very apparent in our system. This result is shown more clearly in Fig. $5(\mathrm{c})$, where we plot the relative magnetization

$$
M(t)=\left[\left|\xi_{+1}(t)\right|^{2}-\left|\xi_{-1}(t)\right|^{2}\right]+\left[\left|\zeta_{+1}(t)\right|^{2}-\left|\zeta_{-1}(t)\right|^{2}\right],
$$

i.e., the population-difference of the states before and after the process $\langle 7\rangle$, as a function of time. It is shown that the central profile of $M(t)$ oscillates around zero with a significant amplitude. In Fig. 6(a) we further show $M(t)$ for various value of interaction intensity $\gamma$. It is shown that, as in the two-body cases, the amplitude and period of the time oscillation of $M(t)$ clearly depends on the value of $\gamma$. Moreover, in our system the time evolution of $M(t)$ also depends on the complex phase factors of the initial state, i.e., $\arg \left[\xi_{m}(t=0)\right]$ and $\arg \left[\zeta_{m}(t=0)\right](m=0, \pm 1)$. It is clear that in our above calculations with initial condition $(49,50)$, we have taken $\arg \left[\zeta_{-1}(t=0)\right]=0.5 \pi$ and $\arg \left[\xi_{0, \pm 1}(t=0)\right]=$ $\arg \left[\zeta_{0,+1}(t=0)\right]=0$. As shown in Fig. $6(\mathrm{~b})$ these initial phase factors are modified, the behavior of $M(t)$ is also seriously changed.

For the single atomic species spinor BEC, the mean field spin dynamics under single-mode-approximation has been mapped to one nonrigid pendulum [58]. The equal-energy contours there being described with two canonical parameters, relative phase and the population fraction, shows that the spin dynamics is very sensitive to the initial states in some parameter regime. For the two atomic species spinor BEC in our manuscript, the system can be mapped to three coupled nonrigid pendulums model [27]. The nonlinear behavior of spin dynamics can be understood by those pendulums models which are charactrized by three pairs of canonical conjugate variables. 


\section{CONCLUSIONS AND DISCUSSIONS}
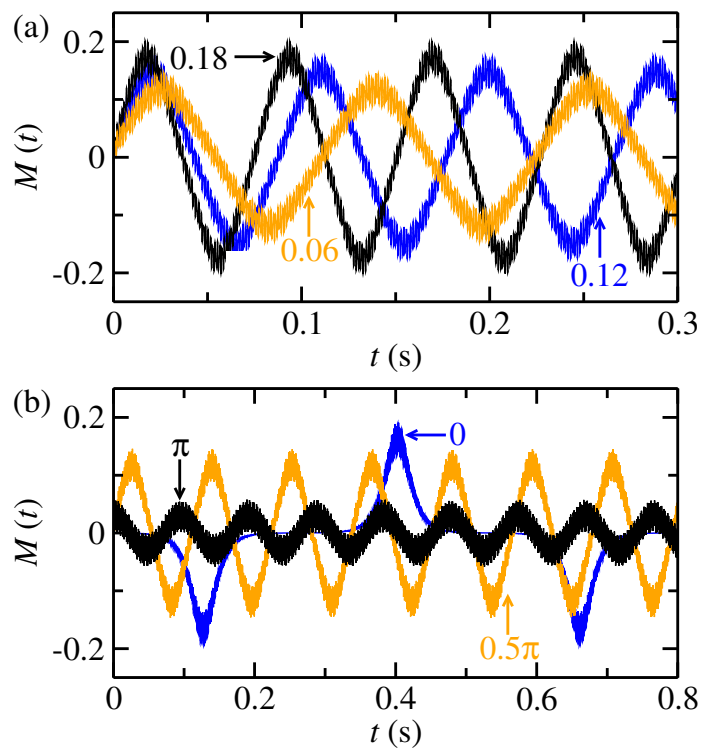

FIG. 6. (Color online) The time evolution of the relative magnetization $M(t)$. (a): Cases with $\gamma=(0.06) \frac{2 \pi \hbar^{2} a_{0}}{\mu}$ (orange line), $\gamma=(0.12) \frac{2 \pi \hbar^{2} a_{0}}{\mu} \pi$ (blue line), and $\gamma=(0.18) \frac{2 \pi \hbar^{2} a_{0}}{\mu}$ (black line). Other parameters are same as Fig. (5). (b): Cases with initial relative phase factor $\arg \left[\zeta_{-1}(t=0)\right]=$ 0 (blue line), $\arg \left[\zeta_{-1}(t=0)\right]=0.5 \pi$ (orange line) and $\arg \left[\zeta_{-1}(t=0)\right]=\pi$ (black line). Other parameters are same as Fig. (5).
In this work we propose an approach for the enhancement and control of the singlet-pairing process between two ultracold spin- 1 atoms of different species, which is based on the combination of the real magnetic field and a laser-induced specie-dependent SMF. Taking the mixture of ultracold ${ }^{87} \mathrm{Rb}$ and ${ }^{23} \mathrm{Na}$ atoms as an example, we illustrate our approach for both a confined two-body system and a two-species spin-1 BEC. It is shown that the singlet-pairing process can be enhanced while the other spin-changing process are suppressed, although the interaction intensity corresponding to the former one is extremely weak. Therefore, our approach can be used for the observation of the singlet-pairing process and the precise measurement of the corresponding interaction parameter, as well as the entanglement generation of two different atoms. Our method also can be applied to other atomic mixture systems in recently experiments, such as ${ }^{7} \mathrm{Li}^{23} \mathrm{Na}$ mixture [10] and ${ }^{7} \mathrm{Li}^{87} \mathrm{Rb}$ mixture [26].

\section{ACKNOWLEDGMENTS}

This work is supported by the National Key R\&D Program of China (Grant No. 2018YFA0306502 (P.Z.)), NSFC Grants No. U1930201(P.Z.) and No. 11674393 (P.Z.).

\section{Appendix A: Derivation of spin dynamic equations in Eq. (44)}

We consider the mixture of BECs of spin- $1{ }^{87} \mathrm{Rb}$ BEC and ${ }^{23} \mathrm{Na}$ BEC atoms with the real magnetic field and the laser induced species-dependent SMF. The many-body Hamiltonian of our system is given by $[28,38]$

$$
\hat{H}=\hat{H}_{\mathrm{Rb}}+\hat{H}_{\mathrm{Na}}+\hat{H}_{\mathrm{Rb}-\mathrm{Na}},
$$

where

$$
\begin{aligned}
\hat{H}_{\mathrm{Rb}} & =\int d \mathbf{r}\left[\hat{\Psi}_{m}^{\dagger}\left(-\frac{\hbar^{2} \nabla^{2}}{2 M_{\mathrm{Rb}}}+V_{\mathrm{Rb}}(\mathbf{r})+E_{m}^{(\mathrm{Rb})}\right) \hat{\Psi}_{m}+\frac{\alpha_{\mathrm{Rb}}}{2} \hat{\Psi}_{i}^{\dagger} \hat{\Psi}_{j}^{\dagger} \hat{\Psi}_{j} \hat{\Psi}_{i}+\frac{\beta_{\mathrm{Rb}}}{2} \hat{\Psi}_{i}^{\dagger} \hat{\Psi}_{k}^{\dagger} F_{\nu, i j} F_{\nu, k l} \hat{\Psi}_{j} \hat{\Psi}_{l}\right], \\
\hat{H}_{\mathrm{Na}} & =\int d \mathbf{r}\left[\hat{\Phi}_{m}^{\dagger}\left(-\frac{\hbar^{2} \nabla^{2}}{2 M_{\mathrm{Na}}}+V_{\mathrm{Na}}(\mathbf{r})+E_{m}^{(\mathrm{Na})}\right) \hat{\Phi}_{m}+\frac{\alpha_{\mathrm{Na}}}{2} \hat{\Phi}_{i}^{\dagger} \hat{\Phi}_{j}^{\dagger} \hat{\Phi}_{j} \hat{\Phi}_{i}+\frac{\beta_{\mathrm{Na}}}{2} \hat{\Phi}_{i}^{\dagger} \hat{\Phi}_{k}^{\dagger} F_{\nu, i j} F_{\nu, k l} \hat{\Phi}_{j} \hat{\Phi}_{l}\right], \\
\hat{H}_{\mathrm{Rb}-\mathrm{Na}} & =\int d \mathbf{r}\left[\alpha \hat{\Psi}_{i}^{\dagger} \hat{\Phi}_{j}^{\dagger} \hat{\Phi}_{j} \hat{\Psi}_{i}+\beta \hat{\Psi}_{i}^{\dagger} \hat{\Phi}_{k}^{\dagger} F_{\nu, i j} F_{\nu, k l} \hat{\Phi}_{l} \hat{\Psi}_{j}+\gamma \frac{(-1)^{i+j}}{3} \hat{\Psi}_{i}^{\dagger} \hat{\Phi}_{-i}^{\dagger} \hat{\Phi}_{-j} \hat{\Psi}_{j}\right] .
\end{aligned}
$$

Here the repeated subscripts means summations for $i, j, k, l, m= \pm 1,0$ and $\nu=x, y, z . \hat{\Psi}_{m}(\mathbf{r})$ and $\hat{\Phi}_{m}(\mathbf{r})(m=0, \pm 1)$ are the annihilation operators of ${ }^{87} \mathrm{Rb}$ and ${ }^{23} \mathrm{Na}$ atom with magnetic quantum number $m$ at position $\mathbf{r} . \quad V_{\mathrm{Rb}}(\mathbf{r})$ and $V_{\mathrm{Na}}(\mathbf{r})$ are the trapping potentials for ${ }^{87} \mathrm{Rb}$ and ${ }^{23} \mathrm{Na}$ atoms, respectively. The interaction strength coefficients $\alpha_{\mathrm{Na}, \mathrm{Rb}}, \beta_{\mathrm{Na}, \mathrm{Rb}}, \alpha, \beta$ and $\gamma$ are defined in Eqs. (3-7), and $F_{\nu=x, y, z}$ are the spin-1 matrices

$$
F_{x}=\frac{1}{\sqrt{2}}\left(\begin{array}{lll}
0 & 1 & 0 \\
1 & 0 & 1 \\
0 & 1 & 0
\end{array}\right), F_{y}=\frac{1}{\sqrt{2}}\left(\begin{array}{ccc}
0 & -\imath & 0 \\
\imath & 0 & -\imath \\
0 & \imath & 0
\end{array}\right), F_{z}=\frac{1}{\sqrt{2}}\left(\begin{array}{ccc}
1 & 0 & 0 \\
0 & 0 & 0 \\
0 & 0 & -1
\end{array}\right) .
$$


Now we apply the mean-field approximation for our system, under which each atom of the same species is in the same one-body state. Furthermore, since in our system the spin-independent interaction intensities (i.e., the $\alpha$-parameters) are much stronger than the spin-dependent interaction intensities (i.e., the $\beta$-parameters and $\gamma$ ), we further use single-mode approximation (except some dynamical mean-field induced resonant regimes [56, 57]) under which the spatial wave function of each atom is spin-independent, and is only determined by the spin-independent interaction. In our calculations based on the above approximations, each ${ }^{87} \mathrm{Rb}$ atom is in the same state corresponding to the wave function

$$
\psi(\mathbf{r})\left[\sum_{m=0, \pm 1} \xi_{m}(t)|m\rangle_{\mathrm{Rb}}\right]
$$

and each ${ }^{23} \mathrm{Na}$ atom is in the same state corresponding to the wave function

$$
\phi(\mathbf{r})\left[\sum_{m=0, \pm 1} \zeta_{m}(t)|m\rangle_{\mathrm{Na}}\right]
$$

with

$$
\int d \mathbf{r}|\psi(\mathbf{r})|^{2}=\int d \mathbf{r}|\phi(\mathbf{r})|^{2}=\sum_{m}\left|\xi_{m}\right|^{2}=\sum_{m}\left|\zeta_{m}\right|^{2}=1 .
$$

Here the spatial wave functions $\psi(\mathbf{r})$ and $\phi(\mathbf{r})$ are determined by the Gross-Pitaevskii equations for the system without the spin-independent interactions:

$$
\begin{gathered}
{\left[-\frac{\hbar^{2} \nabla^{2}}{2 M_{\mathrm{Rb}}}+V_{\mathrm{Rb}}(\mathbf{r})+\alpha_{\mathrm{Rb}} N_{\mathrm{Rb}}|\psi(\mathbf{r})|^{2}+\alpha N_{\mathrm{Na}}|\phi(\mathbf{r})|^{2}\right] \psi(\mathbf{r})=\mu_{\mathrm{Rb}} \psi(\mathbf{r}),} \\
{\left[-\frac{\hbar^{2} \nabla^{2}}{2 M_{\mathrm{Na}}}+V_{\mathrm{Na}}(\mathbf{r})+\alpha_{\mathrm{Na}} N_{\mathrm{Na}}|\phi(\mathbf{r})|^{2}+\alpha N_{\mathrm{Rb}}|\psi(\mathbf{r})|^{2}\right] \phi(\mathbf{r})=\mu_{\mathrm{Na}} \phi(\mathbf{r}),}
\end{gathered}
$$

where $N_{\mathrm{Rb}}$ and $N_{\mathrm{Na}}$ are the numbers of ${ }^{87} \mathrm{Rb}$ and ${ }^{23} \mathrm{Na}$ atoms, respectively, and $\mu_{\mathrm{Rb}}$ and $\mu_{\mathrm{Na}}$ are the corresponding chemical potentials. It is clear that the wave functions (A4, A5) are just the ones in Eq. (39) of Sec. V.

Now we derive the dynamical equation for the coefficients $\xi_{m}(t)$ and $\zeta_{m}(t)(m=0, \pm 1)$. Under the above mean-field and single-mode approximations, the time-dependent many-body state $|\mathrm{Q}(t)\rangle$ of our system can be expressed as

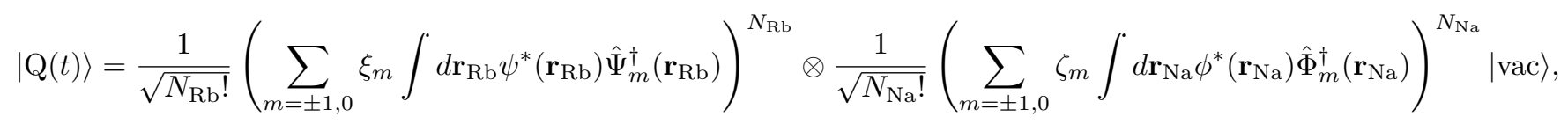

with $|\mathrm{vac}\rangle$ being the vacuum state. Furthermore, the instantaneous average energy of our system on this many-body sate can be expressed as a function of the coefficients $\xi_{m}(t)$ and $\zeta_{m}(t)$, i.e.,

$$
E_{\mathrm{MB}}\left[\xi_{0, \pm 1}(t) ; \xi_{0, \pm 1}^{*}(t) ; \zeta_{0, \pm 1}(t) ; \zeta_{0, \pm 1}^{*}(t)\right] \equiv\langle\mathrm{Q}(t)|\hat{H}| \mathrm{Q}(t)\rangle
$$

Thus, using the time-dependent variational principle, we can obtain the dynamical equations for $\xi_{m}(t)$ and $\zeta_{m}(t)$ $(m=0, \pm 1)$ (up to a global phase factor):

$$
\begin{aligned}
\imath \frac{d}{d t} \xi_{m}(t) & =\frac{\partial E_{\mathrm{MB}}}{\partial \xi_{m}^{*}} \\
\imath \frac{d}{d t} \zeta_{m}(t) & =\frac{\partial E_{\mathrm{MB}}}{\partial \zeta_{m}^{*}}
\end{aligned}
$$

With straightforward calculations, one can find that Eqs. (A11, A12) for $m=0, \pm 1$ are just Eqs. (41-44) in our maintext (up to a global phase factor).

\section{Appendix B: Calculation of atomic probability densities via Thomas-Fermi approximation}

In this appendix we derive the atomic probability densities $|\psi(\mathbf{r})|^{2}$ and $|\phi(\mathbf{r})|^{2}$ via Thomas-Fermi approximation $[59,60]$. Under this approximation, the Gross-Pitaevskii equations in Eq. (A7, A8) can be simplified as

$$
\begin{aligned}
& {\left[V_{\mathrm{Rb}}(\mathbf{r})+\alpha_{\mathrm{Rb}}|\psi(\mathbf{r})|^{2}+\alpha|\phi(\mathbf{r})|^{2}\right] \psi(\mathbf{r})=\mu_{\mathrm{Rb}} \psi(\mathbf{r}),} \\
& {\left[V_{\mathrm{Na}}(\mathbf{r})+\alpha_{\mathrm{Na}}|\phi(\mathbf{r})|^{2}+\alpha|\psi(\mathbf{r})|^{2}\right] \phi(\mathbf{r})=\mu_{\mathrm{Na}} \phi(\mathbf{r}) .}
\end{aligned}
$$


Here we assume the atoms are in the isotropic harmonic trap with the frequency $\omega_{\mathrm{Rb}}$ for ${ }^{87} \mathrm{Rb} \mathrm{BEC}$ and $\omega_{\mathrm{Na}}$ for ${ }^{23} \mathrm{Na}$ $\mathrm{BEC}$, thus the trap potentials are $V_{\mathrm{Rb}, \mathrm{Na}}=M_{\mathrm{Rb}, \mathrm{Na}} \omega_{\mathrm{Rb}, \mathrm{Na}}^{2} r^{2} / 2$. The solutions of these equations are

$$
\begin{aligned}
|\psi(\mathbf{r})|^{2} & =\frac{2 \mu_{\mathrm{Rb}} X_{\mathrm{Rb}}-\omega_{\mathrm{Rb}}^{2} r^{2} Y_{\mathrm{Rb}}}{2 N_{\mathrm{Rb}} Z} \Theta\left(r-r_{\mathrm{tf}, \mathrm{Rb}}\right), \\
|\phi(\mathbf{r})|^{2} & =\frac{2 \mu_{\mathrm{Rb}} X_{\mathrm{Na}}-\omega_{\mathrm{Rb}}^{2} r^{2} Y_{\mathrm{Na}}}{2 N_{\mathrm{Na}} Z} \Theta\left(r-r_{\mathrm{tf}, \mathrm{Na}}\right),
\end{aligned}
$$

where $\Theta(z)$ is the step function which satisfies $\Theta(z)=1$ for $z>0$ and $\Theta(z)=0$ for $z \leq 0, r_{\mathrm{tf}, \mathrm{Rb}}$ and $r_{\mathrm{tf}, \mathrm{Na}}$ are the Tomas-Fermi radius,

$$
r_{\mathrm{tf}, \mathrm{Rb}}=\frac{\sqrt{2 \mu_{\mathrm{Rb}}}}{\omega_{\mathrm{Rb}}} \sqrt{\frac{X_{\mathrm{Rb}}}{Y_{\mathrm{Rb}}}} ; \quad r_{\mathrm{tf}, \mathrm{Na}}=\frac{\lambda_{\omega}}{\sqrt{\lambda_{\mu}}} \frac{\sqrt{2 \mu_{\mathrm{Na}}}}{\omega_{\mathrm{Na}}} \sqrt{\frac{X_{\mathrm{Na}}}{Y_{\mathrm{Na}}}} .
$$

The coefficients $X_{\mathrm{Rb}, \mathrm{Na}}$ are related to the chemical potential ratio $\lambda_{\mu}=\mu_{\mathrm{Na}} / \mu_{\mathrm{Rb}}$,

$$
X_{\mathrm{Rb}}=\lambda_{\mu} \alpha-\alpha_{\mathrm{Na}} ; \quad X_{\mathrm{Na}}=\alpha-\lambda_{\mu} \alpha_{\mathrm{Rb}}
$$

and $Y_{\mathrm{Rb}, \mathrm{Na}}$ are related to the trap frequency ratio $\lambda_{\omega}=\omega_{\mathrm{Na}} / \omega_{\mathrm{Rb}}$,

$$
Y_{\mathrm{Rb}}=\lambda_{\omega}^{2} \alpha M_{\mathrm{Na}}-\alpha_{\mathrm{Na}} M_{\mathrm{Rb}} ; \quad Y_{\mathrm{Na}}=\alpha M_{\mathrm{Rb}}-\lambda_{\omega}^{2} \alpha_{\mathrm{Rb}} M_{\mathrm{Na}} .
$$

$Z, Z=\alpha^{2}-\alpha_{\mathrm{Rb}} \alpha_{\mathrm{Na}}$, is a positive constant coefficient in this system and the chemical potentials $\mu_{\mathrm{Rb}, \mathrm{Na}}$ are determined by the normalization condition (A6).

It is clear that both the probability densities $|\psi(\mathbf{r})|^{2}$ and $|\phi(\mathbf{r})|^{2}$ and the Tomas-Fermi radius $r_{\mathrm{tf}, \mathrm{Rb}}$ and $r_{\mathrm{tf}, \mathrm{Na}}$ should be positive. This yields that the Thomas-Fermi approximation can be used for the systems with $1.998<\omega_{\mathrm{Na}} / \omega_{\mathrm{Rb}}<$ 2.658 and $1.056<\lambda_{\mu}<1.869$.

[1] Y. Kawaguchi and M. Ueda, "Spinor Bose-Einstein condensates," Physics Reports 520, 253 - 381 (2012).

[2] D. M. Stamper-Kurn and M. Ueda, "Spinor Bose gases: Symmetries, magnetism, and quantum dynamics," Rev. Mod. Phys. 85, 1191-1244 (2013).

[3] M. Gabbrielli, Luca Pezzè, and A. Smerzi, "Spin-Mixing Interferometry with Bose-Einstein Condensates," Phys. Rev. Lett. 115, 163002 (2015).

[4] D. Linnemann, H. Strobel, W. Muessel, J. Schulz, R. J. Lewis-Swan, K. V. Kheruntsyan, and M. K. Oberthaler, "Quantum-Enhanced Sensing Based on Time Reversal of Nonlinear Dynamics," Phys. Rev. Lett. 117, 013001 (2016).

[5] S. S. Szigeti, R. J. Lewis-Swan, and S. A. Haine, "Pumped-Up SU(1,1) Interferometry," Phys. Rev. Lett. 118, 150401 (2017).

[6] J. P. Wrubel, A. Schwettmann, D. P. Fahey, Z. Glassman, H. K. Pechkis, P. F. Griffin, R. Barnett, E. Tiesinga, and P. D. Lett, "Spinor Bose-Einstein-condensate phasesensitive amplifier for SU(1,1) interferometry," Phys. Rev. A 98, 023620 (2018).

[7] J. Jie, Q. Guan, and D. Blume, "Spinor Bose-Einstein condensate interferometer within the undepleted pump approximation: Role of the initial state," Phys. Rev. A 100, 043606 (2019).

[8] A. Roulet and C. Bruder, "Synchronizing the Smallest Possible System," Phys. Rev. Lett. 121, 053601 (2018).

[9] A. W. Laskar, P. Adhikary, S. Mondal, P. Katiyar, S. Vinjanampathy, and S. Ghosh, "Observation of Quantum
Phase Synchronization in Spin-1 Atoms," Phys. Rev. Lett. 125, 013601 (2020).

[10] A. Mil, T. V. Zache, A. Hegde, A. Xia, R. P. Bhatt, M. K. Oberthaler, P. Hauke, J. Berges, and F. Jendrzejewski, "A scalable realization of local U(1) gauge invariance in cold atomic mixtures," Science 367, 1128-1130 (2020).

[11] X. Y. Luo, Y. Q. Zou, L. N. Wu, Q. Liu, M. F. Han, M. K. Tey, and L. You, "Deterministic entanglement generation from driving through quantum phase transitions," Science 355, 620-623 (2017).

[12] Y. Q. Zou, L. N. Wu, Q. Liu, X. Y. Luo, S. F. Guo, J. H. Cao, M. K. Tey, and L. You, "Beating the classical precision limit with spin-1 Dicke states of more than 10,000 atoms," Proceedings of the National Academy of Sciences 115, 6381-6385 (2018).

[13] T. Tian, H.-X. Yang, L.-Y. Qiu, H.-Y. Liang, Y.-B. Yang, Y. Xu, and L.-M. Duan, "Observation of Dynamical Quantum Phase Transitions with Correspondence in an Excited State Phase Diagram," Phys. Rev. Lett. 124, 043001 (2020).

[14] M. D. Barrett, J. A. Sauer, and M. S. Chapman, "AllOptical Formation of an Atomic Bose-Einstein Condensate," Phys. Rev. Lett. 87, 010404 (2001).

[15] D. M. Stamper-Kurn, M. R. Andrews, A. P. Chikkatur, S. Inouye, H.-J. Miesner, J. Stenger, and W. Ketterle, "Optical Confinement of a Bose-Einstein Condensate," Phys. Rev. Lett. 80, 2027-2030 (1998).

[16] M. S. Chang, Q. S. Qin, W. X. Zhang, L. You, and M. S. Chapman, "Coherent spinor dynamics in a spin-1 Bose 
condensate," Nature physics 1, 111-116 (2005).

[17] J. Kronjäger, C. Becker, M. Brinkmann, R. Walser, P. Navez, K. Bongs, and K. Sengstock, "Evolution of a spinor condensate: Coherent dynamics, dephasing, and revivals," Phys. Rev. A 72, 063619 (2005).

[18] A. T. Black, E. Gomez, L. D. Turner, S. Jung, and P. D. Lett, "Spinor Dynamics in an Antiferromagnetic Spin-1 Condensate," Phys. Rev. Lett. 99, 070403 (2007).

[19] M. Vengalattore, J. M. Higbie, S. R. Leslie, J. Guzman, L. E. Sadler, and D. M. Stamper-Kurn, "HighResolution Magnetometry with a Spinor Bose-Einstein Condensate," Phys. Rev. Lett. 98, 200801 (2007).

[20] J. Ma, X. Wang, C. P. Sun, and F. Nori, "Quantum spin squeezing," Physics Reports 509, 89-165 (2011).

[21] C. D. Hamley, C. S. Gerving, T. M. Hoang, E. M. Bookjans, and M. S. Chapman, "Spin-nematic squeezed vacuum in a quantum gas," Nature Physics 8, 305 (2012).

[22] L. Pezzè, A. Smerzi, M. K. Oberthaler, R. Schmied, and P. Treutlein, "Quantum metrology with nonclassical states of atomic ensembles," Rev. Mod. Phys. 90, 035005 (2018).

[23] Q. Zhang and A. Schwettmann, "Quantum interferometry with microwave-dressed $F=1$ spinor Bose-Einstein condensates: Role of initial states and long-time evolution," Phys. Rev. A 100, 063637 (2019).

[24] X. Li, B. Zhu, X. He, F. Wang, M. Guo, Z-F Xu, S. Zhang, and D. Wang, "Coherent Heteronuclear Spin Dynamics in an Ultracold Spinor Mixture," Phys. Rev. Lett. 114, 255301 (2015).

[25] L. Li, B. Zhu, B. Lu, S. Zhang, and D. Wang, "Manipulation of heteronuclear spin dynamics with microwave and vector light shift," Phys. Rev. A 101, 053611 (2020).

[26] Fang Fang, Joshua A. Isaacs, Aaron Smull, Katinka Horn, L. Dalila Robledo-De Basabe, Yimeng Wang, Chris H. Greene, and Dan M. Stamper-Kurn, "Collisional spin transfer in an atomic heteronuclear spinor bose gas," Phys. Rev. Research 2, 032054 (2020).

[27] Z. F. Xu, D. J. Wang, and L. You, "Quantum spin mixing in a binary mixture of spin-1 atomic condensates," Phys. Rev. A 86, 013632 (2012).

[28] J-J Chen, Z-F Xu, and L You, "Resonant spin exchange between heteronuclear atoms assisted by periodic driving," Phys. Rev. A 98, 023601 (2018).

[29] M. Luo, Z. Li, and C. Bao, "Bose-Einstein condensate of a mixture of two species of spin-1 atoms," Phys. Rev. A 75, 043609 (2007)

[30] Z. F. Xu, Yunbo Zhang, and L. You, "Binary mixture of spinor atomic Bose-Einstein condensates," Phys. Rev. A 79, 023613 (2009).

[31] Y. Shi, "Ground states of a mixture of two species of spinor Bose gases with interspecies spin exchange," Phys. Rev. A 82, 023603 (2010).

[32] J. Zhang, T. Li, and Y. Zhang, "Interspecies singlet pairing in a mixture of two spin-1 Bose condensates," Phys. Rev. A 83, 023614 (2011).

[33] Z. B. Li, Y. M. Liu, D. X. Yao, and C. G. Bao, "Two types of phase diagrams for two-species Bose-Einstein condensates and the combined effect of the parameters," Journal of Physics B: Atomic, Molecular and Optical Physics 50, 135301 (2017).

[34] Z. F. Xu, Jie Zhang, Yunbo Zhang, and L. You, "Quantum states of a binary mixture of spinor Bose-Einstein condensates," Phys. Rev. A 81, 033603 (2010).

[35] Claude Cohen-Tannoudji and Jacques Dupont-Roc, "Ex- perimental Study of Zeeman Light Shifts in Weak Magnetic Fields," Phys. Rev. A 5, 968-984 (1972).

[36] N. Goldman, G. Juzeliūnas, P. Öhberg, and I. B. Spielman, "Light-induced gauge fields for ultracold atoms," Reports on Progress in Physics 77, 126401 (2014).

[37] S. Knoop, T. Schuster, R. Scelle, A. Trautmann, J. Appmeier, M. K. Oberthaler, E. Tiesinga, and E. Tiemann, "Feshbach spectroscopy and analysis of the interaction potentials of ultracold sodium," Phys. Rev. A 83, 042704 (2011).

[38] F. Wang, D. Xiong, X. Li, D. Wang, and E. Tiemann, "Observation of Feshbach resonances between ultracold $\mathrm{Na}$ and Rb atoms," Phys. Rev. A 87, 050702 (2013).

[39] C. Chin, R. Grimm, P. Julienne, and E. Tiesinga, "Feshbach resonances in ultracold gases," Rev. Mod. Phys. 82, 1225-1286 (2010).

[40] The exact value of $g_{\langle l\rangle}(l=1, \ldots, 7)$ is determined by the detail of the systems. For instance, for the two-atom system of Sec. IV the relevant interaction intensity $g_{\langle 3,4,7\rangle}$ are the non-diagonal elements of the matrix of Eq. (30), with the expressions being given by Eqs. (31-33).

[41] D. A. Steck, "Rubidium 87 D Line Data," (2019).

[42] D. A. Steck, "Sodium D Line Data," (2019).

[43] In experiments one can realize this confinement by using a laser beam with a "magic wavelength" to form the trapping potential, whose corresponding dipole polarizabilities $d_{j}(j=\mathrm{Rb}, \mathrm{Na})$ for each atom satisfy $d_{\mathrm{Rb}} / d_{\mathrm{Na}}=$ $\sqrt{M_{\mathrm{Rb}} / M_{\mathrm{Na}}}$. In this case the trapping potentials $V_{j}$ $(j=\mathrm{Rb}, \mathrm{Na})$ satisfy $V_{\mathrm{Rb}} / V_{\mathrm{Na}}=M_{\mathrm{Rb}} / M_{\mathrm{Na}}$, and thus the trapping frequencies for each atom are same. The "magic wavelength" for $\mathrm{Na}$ and $\mathrm{Rb}$ is approximately $946 \mathrm{~nm}$ [44].

[44] M. S. Safronova, Bindiya Arora, and Charles W. Clark, "Frequency-dependent polarizabilities of alkalimetal atoms from ultraviolet through infrared spectral regions," Phys. Rev. A 73, 022505 (2006).

[45] This conclusion can be obtained via the following analysis. The effect of the transition between the ground state and the $n$-th excited states of $H_{\text {ho }}$ can be estimated via the ratio $\left|D /\left(\varepsilon_{n}-\varepsilon_{0}\right)\right|$, where $\varepsilon_{n}$ and $\varepsilon_{0}$ are the eigenenergies of $H_{\text {ho }}$ corresponding to these two states and $D$ is the corresponding matrix elment of $U_{\mathrm{Rb}-\mathrm{Na}}$. Direct calculation shows that $\left|D /\left(\varepsilon_{n}-\varepsilon_{0}\right)\right| \lesssim\left|a_{\mathrm{Rb}-\mathrm{Na}}^{(0,1,2)} / a_{\mathrm{ho}}\right|$. Thus, when the scattering lengths are much smaller than $a_{\text {ho }}$ we have $\left|D /\left(\varepsilon_{n}-\varepsilon_{0}\right)\right| \ll 1$ which yields the transition can be neglected. ().

[46] In this measurement, if it is required, one can go beyond the approximation that the atomic spatial motion are frozen in the state $|0\rangle_{r}$, and derive the exact solution for the two-body problem with the approach in e.g., the ones in [busch, calarco, chenyue, sala], and compare the exact solution with experimental measurements ().

[47] Q. Guan, V. Klinkhamer, R. Klemt, J. H. Becher, A. Bergschneider, P. M. Preiss, S. Jochim, and D. Blume, "Density Oscillations Induced by Individual Ultracold Two-Body Collisions," Phys. Rev. Lett. 122, 083401 (2019).

[48] Loïc Anderegg, Lawrence W. Cheuk, Yicheng Bao, Sean Burchesky, Wolfgang Ketterle, Kang-Kuen $\mathrm{Ni}$, and John M. Doyle, "An optical tweezer array of ultracold molecules," Science 365, 1156-1158 (2019).

[49] L. R. Liu, J. D. Hood, Y. Yu, J. T. Zhang, N. R. Hutzler, T. Rosenband, and K.-K. Ni, "Building one molecule from a reservoir of two atoms," Science 360, 900-903 
(2018).

[50] M. Höfer, L. Riegger, F. Scazza, C. Hofrichter, D. R. Fernandes, M. M. Parish, J. Levinsen, I. Bloch, and S. Fölling, "Observation of an Orbital InteractionInduced Feshbach Resonance in ${ }^{173}$ Yb," Phys. Rev. Lett. 115, 265302 (2015).

[51] G. Cappellini, L. F. Livi, L. Franchi, D. Tusi, D. Benedicto Orenes, M. Inguscio, J. Catani, and L. Fallani, "Coherent Manipulation of Orbital Feshbach Molecules of Two-Electron Atoms," Phys. Rev. X 9, 011028 (2019).

[52] C. K. Law, H. Pu, and N. P. Bigelow, "Quantum Spins Mixing in Spinor Bose-Einstein Condensates," Phys. Rev. Lett. 81, 5257-5261 (1998).

[53] H. Pu, C. K. Law, S. Raghavan, J. H. Eberly, and N. P. Bigelow, "Spin-mixing dynamics of a spinor BoseEinstein condensate," Phys. Rev. A 60, 1463-1470 (1999).

[54] S. Yi, Ö. E. Müstecaplığlu, C. P. Sun, and L. You, "Single-mode approximation in a spinor-1 atomic condensate," Phys. Rev. A 66, 011601 (2002).

[55] W. X. Zhang, S. Yi, and L. You, "Mean field ground state of a spin-1 condensate in a magnetic field," New Journal of Physics 5, 77-77 (2003).
[56] J. Jie, Q. Guan, S. Zhong, A. Schwettmann, and D. Blume, "Mean-field spin-oscillation dynamics beyond the single-mode approximation for a harmonically trapped spin-1 bose-einstein condensate," Phys. Rev. A 102, 023324 (2020).

[57] J. Jie, S. Zhong, Q. Zhang, I. Morgenstern, H. G. Ooi, Q. Guan, A. Bhagat, D. Nematollahi, A. Schwettmann, and D. Blume, "Dynamical mean-field driven spinor condensate physics beyond the single-mode approximation," submitted for publication.

[58] W. X. Zhang, D. L. Zhou, M.-S. Chang, M. S. Chapman, and L. You, "Coherent spin mixing dynamics in a spin-1 atomic condensate," Phys. Rev. A 72, 013602 (2005).

[59] C. J. Pethick and H. Smith, Bose-Einstein Condensation in Dilute Gases, 2nd ed. (Cambridge University Press, 2008).

[60] F. Wang, X. Li, D. Xiong, and D. Wang, "A double species ${ }^{23} \mathrm{Na}$ and ${ }^{87} \mathrm{Rb}$ Bose-Einstein condensate with tunable miscibility via an interspecies Feshbach resonance," Journal of Physics B: Atomic, Molecular and Optical Physics 49, 015302 (2015). 